\title{
On the importance of nonlinear elastic effects in shear band modeling
}

\author{
Colin McAuliffe * Haim Waisman \\ Department of Civil Engineering and Engineering Mechanics, Columbia University; \\ 610 Seeley W. Mudd Building; 500 West 120th Street, Mail Code 4709; New York, \\ NY 10027
}

\begin{abstract}
Shear bands are typically modeled using grade zero hypoelastic formulations based on an objective stress rate, most often the Jaumann rate. Grade zero hypoelastic formulations have been criticized in the literature as unphysical since it can be shown that such a material is not elastic material defined by a hyperelastic potential, and energy is dissipated around a closed loop of deformation. In addition, the Jaumann rate displays a well known oscillation in large shear. However, numerical studies to date comparing hypo and hyperelastic formulations have shown that these issues are not a concern for metal plasticity under roughly monotonic loading, where elastic strains remain small and there is no accumulation of errors from numerous loading cycles . Numerical implementation of Jaumann rate based formulations is relatively easy, and under certain circumstances leads to symmetric tangent moduli. Due to these three factors, use of the Jaumann rate has persisted despite wellknown theoretical deficiencies. In this work, we compare hyperelastic and Jaumann rate based hypoelastic formulations for shear band modeling, and it is found that early in the deformation, there are no appreciable differences in the two formulations, which is consistent with previous studies reported in the literature. However, later in the deformation, after localization has occurred, the decrease in elastic moduli brings about the nonlinear elastic effects which causes the hyperelastic formulation to lead to more severe localization and more intense plastic straining than the hypoelastic formulation. This occurs even though the maximum principal elastic stretches do not exceed 1.07 in any of the numerical simulations reported in this work. In addition, the effect of elastic nonlinearity is rate dependent, since the rate dependence of plasticity increases the yield stress with increasing strain rate, which leads to larger elastic strains, and more prominent reduction in elastic moduli. Therefore, for shear band modeling, the still popular grade zero hypoelastic formulation based on the Jaumann rate can not be considered a reasonable approximation to the more sound hyperelastic formulation, and should not be used. In light of these results, we may conclude that in addition to the well known fact that use of the Jaumann rate formulation should be constrained to problems with small elastic strains and
\end{abstract}


without cyclical loading, its use should also be constrained to problems where shear banding and localization is not encountered.

\section{Introduction}

Shear banding is a localization phenomenon where thermal softening leads to narrow zones of intense inelastic deformation [Wright, 2002]. While shear bands typically precede ductile fracture, since the thermal softening leads to profound and rapid loss of load carrying capability, this phenomenon is considered a failure mode in its own right [Arriaga et al., 2015, Bai, 1982, Fressengeas and Molinari, 1987, Ling and Belytschko, 2009]

Modeling of shear bands is typically based on a grade zero hypoelastic formulation and the objective Jaumann stress rate, see [Batra and Kim, 1992, Belytschko et al., 1994, Bonnet-Lebouvier et al., 2002, Li et al., 2002, McAuliffe and Waisman, 2014, Nacar et al., 1989, Zhou et al., 1996] among others. Use of this type of formulation in the context of elastoplasticity dates back to [Prager, 1961], but since then has been criticized on theoretical grounds. It can be shown that such a formulation is incompatible with hyperelasticity, meaning that it has not been obtained from a stored energy potential, except under unreasonably restrictive circumstances [Simo and Pister, 1984]. In addition, such a material is not elastic in the sense that the net work around a closed loop is non zero [Bernstein, 1960, Kojić and Bathe, 1987]. These issues are not unique to the Jaumann rate; a grade zero hypoelastic material based on any objective rate except one will not define an elastic material. The one exception is the logarithmic rate which was developed and analyzed in [Bruhns et al., 1999, Xiao et al., 1997a,b, 1999, 2000, Zhu et al., 2014]. This model was shown to be equivalent to the rate form of the hyperelastic model based on the logarithmic strain, and is the only rate among infinitely many possibilities for which this is true.

Several hyperelasticty based plasticity models have been developed using Neo Hookean energy potentials [Simo, 1988, Simo and Hughes, 1998, Simo and Miehe, 1992], and hyperelastic potentials based on logarithmic strain measures [Auricchio and Taylor, 1999, Eterovic and Bathe, 1990, Weber and Anand, 1990]. These models define truly elastic materials, and objective stress rates are not needed since objectivity of the elastic relations are automatically satisfied. Despite this, grade zero hypoelastic formulations remain quite popular since for metal plasticity, the assumption of constant elastic moduli is generally

\footnotetext{
* Corresponding author: cjm2176@columbia.edu (Colin McAuliffe)
} 
appropriate because elastic stretches often remain small. This has been borne out by numerical comparisons of hyper and grade zero hypoelastic formulations for simulating various plasticity problems [Anand, 1979, Brepols et al., 2014], which show only minor differences between the two. Comparisons such as this have been used to justify the continued use of grade zero hypoelasticity for plasticity modeling. In addition, referring specifically to Jaumann rate based formulations, a symmetric tangent modulus can be obtained for numerical computations for certain problems, which provides further motivation for use of this formulation.

However, these studies did not examine problems which lead to severe localization, and while hyperelastic models have been used to simulate localization in granular materials[Andrade and Borja, 2006, Borja, 2002, Borja et al., 2013] as well as rubber - like materials[Triantafyllidis and Aifantis, 1986]and general hyperelastic materials[Leroy and Molinari, 1993], to our knowledge the role of nonlinear elasticity in the formation of shear bands have not been studied. In the numerical results shown below, it is found that while the maximum principal elastic stretches remain below 1.07, the effects of nonlinear elasticity lead to significant differences in observed behavior when compared to the often used Jaumann rate based grade zero hypoelastic model. The reason for the marked differences between the formulations found here, in contrast to the minor differences reported in previous work is that the nonlinear elastic effects due to the hyperelastic material law do not play a significant role until the later stages in the deformation. Nonlinear elasticity tends to increase the extent of the deformation after localization has occurred, since the material is more compliant due to the reduction in elastic moduli. This occurs even though the reduction in moduli is fairly small. In addition, the spurious elastic energy dissipation produced by hypoelastic formulations leads to an unphysical increase in the energy dissipation capacity of the material. Since the Jaumann rate based formulation is the most commonly used for shear band modeling, we have only shown results for this one objective rate. However, with the unique exception of the logarithmic rate, qualitatively the above conclusions would not be affected by the choice of objective rate. Therefore, for shear band modeling, a grade zero hypoelastic formulation with the Jaumann rate can not be considered a reasonable approximation to the more sound hyperelastic formulation, and should not be used. Hyperelastic formulations, represented either in total form, or rate form, should be used instead.

Secondly, since the shear band model involves nonsymmetric thermo mechanical coupling, a symmetric tangent for the global system can not be obtained regardless of the elasticity model chosen. This negates the numerical advantage of using the popular Jaumann rate based formulation. It is shown that this behavior is important for applications where the energy absorption of the material is of interest, since the hyperelastic formulation predicts less dissipation than the hypoelastic formulation. While the model here is for metals with ther- 
mal softening shear bands, we expect that similar trends would be observed if a comparison of hyper vs hypoelasticity were undertaken for other shear bands models such as gradient plasticity [Anand et al., 2005, 2012, Gurtin and Anand, 2005].

The role of the nonlinear elastic effect increases with an increase in strain rate, since the rate dependence of plasticity delays yielding for higher strain rates, which leads to larger elastic strains and greater reduction of the elastic moduli. This is of interest in the ductile - brittle failure transition, which is governed by the competition between the formation of shear bands due to thermal softening, and the development of a crack due to large principal stress [Needleman and Tvergaard, 1995, 2000]. Hyperelasticity will affect both aspects of the transition since as shown here, the nonlinear elastic softening accelerates the formation of a shear band, and as shown by [Buehler et al., 2003], nonlinear elastic softening decrease the energy flow to the crack tip. Thus, in comparison to a linear elastic solid, an elastic softening material will shear band more readily and fracture less readily. For a material that stiffens elastically, the opposite behavior is expected.

The paper is organized as follows: in section 2 the kinematics and inelastic constitutive relations are developed. We emphasize that these aspects are the same for both the grade zero hypoelastic and hyperelastic formulations. In section 3, the two formulations depart as the elastic constitutive relations are specified, and the numerics for each formulation are developed. Numerical results for a few example problems, are shown in section 4 .

Throughout this work, quantities that are associated with the current configuration are represented by lower case letters with lower case subscripts i, j, k, and 1 , for example $d_{i j}$. For quantities associated with the reference configuration, capital letters are used with capital subscripts A, B, C, D, for example $C_{A B}$. For the intermediate configuration defined by the multiplicative split of the deformation gradient, hatted lower case letters are used, with lower case

subscripts a, b, c, d, such as $\hat{d}_{a b}$. Tensor quantities are denoted by bold face, while scalars and tensor components use regular faced type.

\section{Kinematics and inelastic constitutive relations}

The basic kinematic quantities and inelastic constitutive relations that are used in this work are defined in this section. For the rate based formulation the most important kinematic quantity is the elastic rate of deformation $\mathbf{d}^{e}$ while the most important quantity for the hyperelastic formulation is the elastic left Cauchy Green tensor $\mathbf{b}^{e}$. However, both models employ the same kinematic framework and the same inelastic constitutive relation. 


\subsection{Kinematics}

A multiplicative decomposition of the deformation gradient $\mathbf{F}$ is assumed (see also Figure 1) so that

$$
F_{i A}=F_{i a}^{e} F_{a q}^{t} F_{q A}^{p}
$$

Where elastic deformations are included in $\mathbf{F}^{e}$, thermal deformations in $\mathbf{F}^{t}$ and irreversible, inelastic deformations are in $\mathbf{F}^{p}$. The determinant of the deformation gradient describes the local volume change, and is often called the Jacobian

$$
J=\operatorname{det}(\mathbf{F})=\operatorname{det}\left(\mathbf{F}^{e}\right) \operatorname{det}\left(\mathbf{F}^{t}\right) \operatorname{det}\left(\mathbf{F}^{p}\right)=J^{e} J^{t} J^{p}
$$

In this work, isochoric metal plasticity is assumed so that $J^{p}=1$. With the multiplicative split, the velocity gradient $\mathbf{l}$, which is defined as

$$
l_{i j}=\dot{F}_{i A} F_{A j}^{-1}
$$

can thus be expanded as

$$
l_{i j}=\dot{F}_{i a}^{e} F_{a j}^{e,-1}+F_{i a}^{e} \dot{F}_{a q}^{t} F_{q b}^{t,-1} F_{b j}^{e,-1}+F_{i a}^{e} F_{a q}^{t} \dot{F}_{q A}^{p} F_{A r}^{p,-1} F_{r b}^{t,-1} F_{b j}^{e,-1}
$$

This can be viewed as an additive split of the velocity gradient, where the first term on the right hand side of (4) is the elastic part of 1 , the second term is the thermal part and the third is the inelastic part, i.e.

$$
\begin{aligned}
l_{i j}^{e} & =\dot{F}_{i a}^{e} F_{a j}^{e,-1} \\
l_{i j}^{t} & =F_{i a}^{e} \dot{F}_{a q}^{t} F_{q b}^{t,-1} F_{b j}^{e,-1} \\
l_{i j}^{p} & =F_{i a}^{e} F_{a q}^{t} \dot{F}_{q A}^{p} F_{A r}^{p,-1} F_{r b}^{t,-1} F_{b j}^{e,-1}
\end{aligned}
$$

Isotropic thermal expansion is considered, allowing for simplification of the thermal and inelastic velocity gradients in equations (6) and (7). The thermal deformation gradient and its time derivative are

$$
\begin{aligned}
& F_{i q}^{t}=\aleph(T) \delta_{i q} \\
& \dot{F}_{i q}^{t}=\frac{\partial \aleph}{\partial T} \dot{T} \delta_{i q}
\end{aligned}
$$

Where $\aleph(T)$ is the thermal stretch [Lubarda, 2004]. Then the quantity $\dot{F}_{a q}^{t} F_{q b}^{t,-1}=$ $\alpha \dot{T} \delta_{a b}$ where the coefficient of thermal expansion is given by $\alpha=\frac{1}{\aleph} \frac{\partial \aleph}{\partial T}$. The thermal and plastic velocity gradients are now

$$
\begin{aligned}
& l_{i j}^{t}=\alpha \dot{T} \delta_{i j} \\
& l_{i j}^{p}=F_{i q}^{e} \dot{F}_{q A}^{p} F_{A r}^{p,-1} F_{r j}^{e,-1}
\end{aligned}
$$


and the total deformation gradient becomes

$$
F_{i A}=\aleph F_{i q}^{e} F_{q A}^{p}
$$

The symmetric part of the velocity gradient is known as the rate of deformation d, namely

$$
d_{i j}=\operatorname{sym}\left(l_{i j}\right)=\frac{1}{2}\left(l_{i j}+l_{j i}\right)
$$

This can also be decomposed additively into elastic, thermal, and plastic parts as follows

$$
\begin{aligned}
d_{i j}^{e} & =\frac{1}{2}\left(\dot{F}_{i a}^{e} F_{a j}^{e,-1}+\dot{F}_{j a}^{e} F_{a i}^{e,-1}\right) \\
d_{i j}^{t} & =\alpha \dot{T} \delta_{i j} \\
d_{i j}^{p} & =\frac{1}{2}\left(F_{i q}^{e} \hat{l}_{q r}^{p} F_{r j}^{e,-1}+F_{j q}^{e} \hat{l}_{q r}^{p} F_{r i}^{e,-1}\right)
\end{aligned}
$$

where the plastic velocity gradient with respect to the intermediate configuration $\hat{\mathbf{l}}^{p}$ is given by

$$
\hat{l}_{q r}^{p}=\dot{F}_{q A}^{p} F_{A r}^{p,-1}
$$

This tensor will be fully specified later in section 2.2 , where the inelastic constitutive relations are defined. Once specified, the elastic rate of deformation $\mathbf{d}^{e}$ can be defined in terms of the total, thermal and plastic rates of deformation as follows

$$
d_{i j}^{e}=d_{i j}-d_{i j}^{p}-d_{i j}^{t}
$$

This is the primary kinematic quantity used to define the rate form elastic constitutive relation.

For the hyperelastic form, the primary kinematic quantity is the elastic left Cauchy Green tensor $\mathbf{b}^{e}$. This is

$$
b_{i j}^{e}=F_{i q}^{e} F_{j q}^{e}
$$


The elastic deformation gradient can be found by solving (12) and is given by

$$
F_{i q}^{e}=\frac{1}{\aleph} F_{i A} F_{A q}^{p,-1}
$$

Substituting this into (19) along with the definition of the thermal deformation gradient given by (8) gives

$$
b_{i j}^{e}=F_{i A} C_{A B}^{t p,-1} F_{j B}
$$

where

$$
C_{A B}^{t p,-1}=\frac{1}{\aleph^{2}} F_{A q}^{p,-1} F_{B q}^{p,-1}
$$

This is the inverse of the thermal-plastic right Cauchy Green tensor.

One of the hyperelastic models employed here is based on the so called Hencky strain.[Hencky, 1928] This strain measure is the natural logarithm of the left elastic stretch $\mathbf{v}^{e}=\sqrt{\mathbf{b}^{e}}$, and is defined as

$$
\boldsymbol{v}^{e}=\ln \left(\mathbf{v}^{e}\right)=\sum_{A=1}^{3} \ln \left(\epsilon_{A}^{e}\right) p_{A} \otimes p_{A}
$$

Where the principal elastic stretches are the $\left(\epsilon_{A}^{e}\right)$ and $p_{A}$ represent the principal directions with respect to the deformed configuration. Since $J^{e}=\epsilon_{1}^{e} \epsilon_{2}^{e} \epsilon_{3}^{e}$ then we can write

$$
\begin{aligned}
\ln \left(J^{e}\right) & =\ln \left(\epsilon_{1}^{e} \epsilon_{2}^{e} \epsilon_{3}^{e}\right) \\
& =\ln \left(\epsilon_{1}^{e}\right)+\ln \left(\epsilon_{2}^{e}\right)+\ln \left(\epsilon_{3}^{e}\right) \\
& =v_{k k}^{e}
\end{aligned}
$$

and therefore, the logarithm of the elastic Jacobian is the trace of the logarithmic elastic strain.

Some additional kinematic quantities will be needed for section 3, which will be defined here and recalled later. The elastic Right Cauchy Green tensor $\mathbf{C}^{e}$, is defined as

$$
C_{q r}^{e}=F_{k q}^{e} F_{k r}^{e}
$$

giving the elastic Green strain $\mathbf{E}^{e}$ as

$$
E_{q r}^{e}=\frac{1}{2}\left(C_{q r}^{e}-\delta_{q r}\right)
$$


Differentiating (28) with respect to time gives the following expression for the rate of Green strain

$$
\dot{E}_{q r}^{e}=\frac{1}{2}\left(\dot{F}_{k q}^{e} F_{k r}^{e}+F_{k q}^{e} \dot{F}_{k r}^{e}\right)
$$

Note that the first and second terms of this equation can be written respectively as

$$
\begin{aligned}
& \dot{F}_{k q}^{e} F_{k r}^{e}=F_{i q}^{e} \dot{F}_{j a}^{e} F_{a i}^{e,-1} F_{j r}^{e}=F_{i q}^{e} l_{j i}^{e} F_{j r}^{e} \\
& F_{k q}^{e} \dot{F}_{k r}^{e}=F_{i q}^{e} \dot{F}_{i a}^{e} F_{a j}^{e,-1} F_{j r}^{e}=F_{i q}^{e} l_{i j}^{e} F_{j r}^{e}
\end{aligned}
$$

and thus the elastic deformation rate and the rate of elastic Green strain have the relation

$$
\dot{E}_{q r}^{e}=\frac{1}{2} F_{i q}^{e}\left(l_{j i}^{e}+l_{i j}^{e}\right) F_{j r}^{e}=F_{i q}^{e} d_{i j}^{e} F_{j r}^{e}
$$

\subsection{Inelastic Constitutive Relations}

The equivalent plastic strain $\bar{\gamma}^{p}$ evolves according to the following equation

$$
\dot{\bar{\gamma}}^{p}=\sqrt{\frac{2}{3} d_{i j}^{p} d_{i j}^{p}}
$$

To specify $\mathbf{l}^{p}$ and hence $\mathbf{d}^{p}$, first the symmetric part of $\hat{\mathbf{l}}^{p}$ is denoted by $\hat{\mathbf{d}}^{p}$ and defined as [Lubarda, 2004, Miehe, 1994, Simo and Miehe, 1992]

$$
\hat{d}_{q r}^{p}=\frac{1}{2}\left(\hat{l}_{q r}^{p}+\hat{l}_{r q}^{p}\right)=g\left(\bar{\sigma}, T, \bar{\gamma}^{p}\right) \hat{n}_{q s} C_{s r}^{e}
$$

Where $g\left(\bar{\sigma}, T, \bar{\gamma}^{p}\right)$ is a constitutive function with units of strain rate and $\hat{n}_{q s}$ is the direction of plastic flow with respect to the intermediate configuration. The kinematic decomposition given by (1) only defines the intermediate configuration up to a rigid body rotation. However, rigid body rotations on the intermediate configuration have no effect upon the elastic constitutive laws given in section 3 . Thus the antisymmetric part of $\hat{\mathbf{l}}^{p}$ can be chosen arbitrarily, as long as all other constitutive equations are consistent with this choice [Dafalias, 1998]. For convenience, the so called spinless configuration is chosen so that $\hat{l}_{q r}^{p}=\hat{l}_{r q}^{p}$, and thus plugging (34) into (11) gives

$$
\begin{aligned}
l_{i j}^{p} & =g\left(\bar{\sigma}, T, \bar{\gamma}^{p}\right) F_{i q}^{e} \hat{n}_{q s} C_{s r}^{e} F_{r j}^{e,-1} \\
& =g\left(\bar{\sigma}, T, \bar{\gamma}^{p}\right) n_{i j}
\end{aligned}
$$

Where the plastic flow direction with respect to the current configuration is $n_{i j}=\hat{n}_{q r} F_{i q}^{e} F_{j r}^{e}$ is defined as

$$
n_{i j}=\frac{3 s_{i j}}{2 \bar{\sigma}}
$$


where $s_{i j}=\sigma_{i j}-\frac{1}{3} \sigma_{k k} \delta_{i j}$ is the deviatoric Cauchy stress and $\bar{\sigma}=\sqrt{\frac{3}{2} s_{i j} s_{i j}}$ is the effective Cauchy stress. Since $\mathbf{n}$ is symmetric, then $\hat{\mathbf{l}}^{p}=\hat{\mathbf{d}}^{p}$ and

$$
d_{i j}^{p}=g\left(\bar{\sigma}, T, \bar{\gamma}^{p}\right) n_{i j}
$$

Using (37) and (36) in (33) gives

$$
\dot{\bar{\gamma}}^{p}=g\left(\bar{\sigma}, T, \bar{\gamma}^{p}\right)
$$

and thus the constitutive function $g\left(\bar{\sigma}, T, \bar{\gamma}^{p}\right)$ gives the equivalent plastic strain rate as a function of stress, temperature and equivalent plastic strain. Since the thermal and plastic velocity gradients are symmetric, the total material spin is contained in the elastic velocity gradient, i.e.

$$
\omega_{i j}=\operatorname{asym}(\mathbf{l})=\operatorname{asym}\left(\mathbf{l}^{e}\right)=\frac{1}{2}\left(l_{i j}-l_{j i}\right)
$$

and the additive decomposition of the velocity gradient (4) becomes

$$
l_{i j}=l_{i j}^{e}+d_{i j}^{t}+d_{i j}^{p}
$$

where

$$
l_{i j}^{e}=d_{i j}^{e}+\omega_{i j}
$$

\subsection{Update equation for the left Cauchy Green tensor}

In the numerical section of this work, $\mathbf{b}^{e}$ will be treated as a history variable and updated incrementally. Here we develop the strong form for the update equation for $\mathbf{b}^{e}$ in terms of the thermal and inelastic deformation rates given in the preceding section. First, note that since $\mathbf{b}^{e}$ is defined as the push forward of $\dot{\mathbf{C}}^{t p,-1}$, then the Lie derivative of $\mathbf{b}^{e}$ is

$$
£_{v} b_{i j}^{e}=F_{i A} \dot{C}_{A B}^{t p,-1} F_{j B}
$$

$\dot{\mathbf{C}}^{t p,-1}$ is found by taking the time derivative of equation (22), and leads to the following

$$
\dot{C}_{A B}^{t p,-1}=\frac{1}{\aleph^{2}}\left[\dot{F}_{A q}^{p,-1} F_{B q}^{p,-1}+F_{A q}^{p,-1} \dot{F}_{B q}^{p,-1}-2 \alpha \dot{T} F_{A q}^{p,-1} F_{B q}^{p,-1}\right]
$$

Then plugging this along with equation (12) into (43) leads to

$$
£_{v} b_{i j}^{e}=F_{i r}^{e}\left\{F_{r A}^{p} \dot{F}_{A s}^{p,-1}+F_{s A}^{p} \dot{F}_{A r}^{p,-1}-2 \alpha \dot{T} \delta_{r s}\right\} F_{j s}^{e}
$$


Using the identity for the derivative of the inverse of a tensor

$$
\dot{F}_{A s}^{p,-1}=-F_{A q}^{p,-1} \dot{F}_{q C}^{p} F_{C s}^{p,-1}
$$

then plugging (46) into (45)

$$
\begin{aligned}
-\frac{1}{2} £_{v} b_{i j}^{e} & =F_{i r}^{e}\left\{\hat{d}_{r s}^{p}+\alpha \dot{T} \delta_{r s}\right\} F_{j s}^{e} \\
& =F_{i r}^{e} \hat{d}_{r s}^{p} F_{j s}^{e}+\alpha \dot{T} b_{i j}^{e}
\end{aligned}
$$

Lastly, both sides are multiplied by $b_{j k}^{e,-1} b_{k l}^{e}=\delta_{j l}$

$$
\begin{aligned}
-\frac{1}{2} £_{v} b_{i l}^{e} & =\left(F_{i r}^{e} \hat{d}_{r s}^{p} F_{s k}^{e,-1}+\alpha \dot{T} \delta_{i k}\right) b_{k l}^{e} \\
& =\left(d_{i k}^{p}+d_{i k}^{t}\right) b_{k l}^{e}
\end{aligned}
$$

Note that from equation (15), $\alpha \dot{T} \delta_{i k}$ is the thermal rate of deformation. Similarly, since $\hat{\mathbf{l}}^{p}$ and $\mathbf{l}^{p}$ have been defined to be symmetric in the previous section, $F_{i r}^{e} \hat{d}_{r s}^{p} F_{s k}^{e,-1}$ is the plastic rate of deformation with respect to the current configuration $\mathbf{d}^{p}$. Substituting (36) gives

$$
-\frac{1}{2} £_{v} b_{i j}^{e}=\left[g\left(\bar{\sigma}, T, \bar{\gamma}^{p}\right) n_{i k}+\alpha \dot{T} \delta_{i k}\right] b_{k j}^{e}
$$

which will form the basis for the incremental update of $\mathbf{b}^{e}$ developed in later sections. There is no need for such an update in the rate form constitutive law which is written in terms of $\mathbf{d}^{e}$ and computed directly from (18). Up until this point, the kinematic assumptions and thermal and inelastic constitutive laws for both the rate and total forms are the same. In the next section, where the elastic constitutive relations are defined, the two forms will depart from one another.

\section{Elastic Constitutive Relations}

The elastic constitutive relations begin with the hyperelastic relation

$$
\hat{s}_{q r}^{e}=2 \frac{\partial \phi}{\partial C_{q r}^{e}}
$$


Where $\hat{\mathbf{s}}^{e}$ is the second Piola-Kirchhoff stress on the intermediate configuration, and $\phi$ is the hyperelastic potential. The Kirchhoff $\boldsymbol{\tau}$ stress is obtained by transforming this equation to the current configuration with the elastic deformation gradient $\mathbf{F}^{e}$ so that

$$
\tau_{i j}=F_{i q}^{e} F_{j r}^{e} \hat{s}_{q r}^{e}
$$

The rate form constitutive equations are developed by first taking the material time derivative of (53), leading to

$$
\dot{\tau}_{i j}=F_{i q}^{e} F_{j r}^{e} \dot{\hat{s}}_{q r}^{e}+\dot{F}_{i q}^{e} F_{j r}^{e} \hat{s}_{q r}^{e}+F_{i q}^{e} \dot{F}_{j r}^{e} \hat{s}_{q r}^{e}
$$

The second and third terms may be written as

$$
\begin{aligned}
& \dot{F}_{i q}^{e} F_{j r}^{e} \hat{s}_{q r}^{e}=\dot{F}_{i q}^{e} F_{r k}^{e,-1} F_{k a}^{e} F_{j r}^{e} \hat{s}_{a r}^{e}=l_{i k}^{e} \tau_{k j} \\
& F_{i q}^{e} \dot{F}_{j r}^{e} \hat{s}_{q r}^{e}=F_{i q}^{e} \dot{F}_{j r}^{e} F_{r k}^{e,-1} F_{k a}^{e} \hat{s}_{a r}^{e}=\tau_{i k} l_{j k}^{e}
\end{aligned}
$$

and thus (54) may be written as

$$
\dot{\tau}_{i j}=£_{v}^{e} \tau_{i j}+l_{i k}^{e} \tau_{k j}+\tau_{i k} l_{j k}^{e}
$$

where the elastic Lie derivative of the Kirchhoff stress is

$$
\begin{aligned}
£_{v}^{e} \tau_{i j} & =F_{i q}^{e} F_{j r}^{e} \dot{\hat{s}}_{q r}^{e} \\
& =\dot{\tau}_{i j}-l_{i k}^{e} \tau_{k j}-\tau_{i k} l_{j k}^{e}
\end{aligned}
$$

In the numerical development in the following section, expressions in terms of the total Lie derivative of the Kirchhoff stress $£_{v} \tau_{i j}=\dot{\tau}_{i j}-l_{i k} \tau_{k j}-\tau_{i k} l_{j k}$ are preferred. Substituting the decomposition of $\mathbf{l}$ given by (41), $£_{v} \boldsymbol{\tau}$ becomes

$$
£_{v} \tau_{i j}=\dot{\tau}_{i j}-\left(l_{i k}^{e}+d_{i k}^{t}+d_{i k}^{p}\right) \tau_{k j}-\tau_{i k}\left(l_{j k}^{e}+d_{j k}^{t}+d_{j k}^{p}\right)
$$

Comparing this to equation (59) allows $£_{v} \boldsymbol{\tau}$ to be written in terms of $£_{v}^{e} \boldsymbol{\tau}$ as follows

$$
£_{v} \tau_{i j}=£_{v}^{e} \tau_{i j}-d_{i k}^{t p} \tau_{k j}-\tau_{i k} d_{j k}^{t p}
$$

where $d_{i k}^{t p}$ is short hand for $d_{i k}^{t}+d_{i k}^{p}$.

Using (32) with (58) and combining the result with (61) leads to an expression entirely in terms of deformed configuration quantities as follows

$$
£_{v} \tau_{i j}=c_{i j k l}^{e l a s} d_{k l}^{e}-d_{i k}^{t p} \tau_{k j}-\tau_{i k} d_{j k}^{t p}
$$


where

$$
c_{i j k l}^{e l a s}=4 F_{i q}^{e} F_{j r}^{e} F_{k s}^{e} F_{l t}^{e} \frac{\partial^{2} \phi}{\partial C_{q r}^{e} \partial C_{s t}^{e}}
$$

This is the general rate form of a hyperelastic law under the kinematic framework and the plastic and thermal constitutive assumptions of the previous section.

\subsection{Hyperelastic Models}

Several hyperelasticty based plasticity models have been developed using Neo Hookean energy potentials [Simo, 1988, Simo and Hughes, 1998, Simo and Miehe, 1992], and hyperelastic potentials based on the Hencky strain [Auricchio and Taylor, 1999, Eterovic and Bathe, 1990, Weber and Anand, 1990]. Aside from differences in the chosen hyperelastic potentials, the update of the elastic strain measures may be done in different ways. One method, used in [Eterovic and Bathe, 1990, Idesman, 2003, Weber and Anand, 1990], is to use (11) and (36) to obtain an update equation for the inelastic deformation gradient

$$
\dot{F}_{q A}^{p}=g\left(\bar{\sigma}, T, \bar{\gamma}^{p}\right) \hat{n}_{q s} C_{s r}^{e} F_{r A}^{p}
$$

Upon numerical integration of this equation, the elastic deformation gradient is computed from (12) and other elastic strain measures can be computed from the elastic deformation gradient as needed. The second method, used by [Auricchio and Taylor, 1999, Simo, 1988, Simo and Hughes, 1998, Simo and Miehe, 1992], is to update the left elastic Cauchy Green tensor by integrating an equation similar to (51). From a theoretical viewpoint, these groups of models are equivalent in the sense that it can be shown that the flow rules (51) and (64) are equivalent [Idesman, 2003]. However while the flow rules are equivalent on the continuous level, differences may arise due to the integration procedure employed for numerical computations.

Here, we obtain results from both the Neo Hookean, and Hencky strain based models. Both models use $\mathbf{b}^{e}$ as a strain measure, and the numerical update of this tensor is identical for each model. This update procedure is described first, and later, the specific forms of the two hyperelastic models are given.

The update equations for the left elastic Cauchy green tensor are obtained following the procedure given in [Simo and Hughes, 1998], and begins with equation (51)

$$
£_{v} b_{i j}^{e}=F_{i A} \dot{C}_{A B}^{t p,-1} F_{j B}=-2\left[g\left(\bar{\sigma}, T, \bar{\gamma}^{p}\right) n_{i k}+\alpha \dot{T} \delta_{i k}\right] b_{k j}^{e}
$$


This equation can be simplified by utilizing the fact that the yield stress in metals is significantly smaller than the elastic modulus. This follows from splitting $\mathbf{b}^{e}$ into volumetric and deviatoric parts, and substituting the definitions of $\mathbf{d}^{p}$ and $\overline{\mathbf{b}}^{e}$ from equations (38) and (86) respectively

$$
-2 d_{i k}^{p} b_{k j}^{e}=-2 g\left(\bar{\sigma}, T, \bar{\gamma}^{p}\right) n_{i k} J^{e, 2 / 3}\left[\frac{1}{3} \delta_{k j} \bar{b}_{l l}^{e}+\operatorname{dev}\left(\bar{b}_{k j}^{e}\right)\right]
$$

Substituting the equations (37) and (88), and noting that $\bar{\tau}=J \bar{\sigma}$ and $\operatorname{dev}\left(\tau_{i j}\right)=$ $J s_{i j}$, this becomes

$$
-2 d_{i k}^{p} b_{k j}^{e}=-3 g\left(\bar{\sigma}, T, \bar{\gamma}^{p}\right) J^{e, 2 / 3}\left[\frac{1}{3} \frac{\operatorname{dev}\left(\tau_{i j}\right)}{\bar{\tau}} \bar{b}_{k k}^{e}+\frac{\operatorname{dev}\left(\tau_{i k}\right)}{\bar{\tau}} \frac{\operatorname{dev}\left(\tau_{k j}\right)}{\mu}\right]
$$

The second term in brackets can be written as

$$
\frac{\operatorname{dev}\left(\tau_{i k}\right)}{\bar{\tau}} \frac{\operatorname{dev}\left(\tau_{k j}\right)}{\mu}=\frac{\operatorname{dev}\left(\tau_{i k}\right)}{\bar{\tau}} \frac{\operatorname{dev}\left(\tau_{k j}\right)}{\bar{\tau}} \frac{\bar{\tau}}{\mu}
$$

The magnitude of $\frac{\operatorname{dev}\left(\tau_{k j}\right)}{\bar{\tau}}$ is 1 and effective stress is a few orders of magnitude less than the shear modulus for metals. Therefore, this term can safely be neglected.

With this assumption, (65) becomes

$$
£_{v} b_{i j}^{e}=F_{i A} \dot{C}_{A B}^{t p,-1} F_{j B} \approx-\frac{2}{3} g\left(\bar{\sigma}, T, \bar{\gamma}^{p}\right) n_{i j} b_{k k}^{e}-2 \alpha \dot{T} b_{i j}^{e}
$$

This expression is pulled back to the undeformed configuration as follows

$$
\dot{C}_{A B}^{t p,-1}=-2 F_{A i}^{-1} F_{B j}^{-1}\left(\frac{1}{3} g\left(\bar{\sigma}, T, \bar{\gamma}^{p}\right) n_{i j} b_{k k}^{e}+\alpha \dot{T} b_{i j}^{e}\right)
$$

The backward Euler method is employed to discretize this rate equation for $\mathbf{C}^{t p,-1}$

${ }_{n+1} C_{A B}^{t p,-1}-{ }_{n} C_{A B}^{t p,-1}=-2 \Delta t_{n+1} F_{A i n+1}^{-1} F_{B j}^{-1}\left(\frac{1}{3}{ }_{n+1} g\left(\bar{\sigma}, T, \bar{\gamma}^{p}\right){ }_{n+1} n_{i j n+1} b_{k k}^{e}+\alpha_{n+1} \dot{T}_{n+1} b_{i j}^{e}\right)$

The update equation for $\mathbf{b}^{e}$ is then obtained by pushing (71) to the current configuration and solving for $\mathbf{b}^{e}$, resulting in

$$
{ }_{n+1} b_{i j}^{e}=\theta\left[\tilde{b}_{i j}^{e}-\frac{2}{3} \Delta t_{n+1} g\left(\bar{\sigma}, T, \bar{\gamma}^{p}\right){ }_{n+1} n_{i j}{ }_{n+1} b_{k k}^{e}\right]
$$


where the thermal expansion contribution is captured by $\theta=\frac{1}{1+2 \Delta t \alpha_{n+1} \dot{T}}$ and $\tilde{\mathbf{b}}^{e}$ is $\mathbf{C}^{t p,-1}$ at time step $\mathrm{n}$, pushed to the current configuration at time step $\mathrm{n}+1$, i.e.

$$
\tilde{b}_{i j}^{e}={ }_{n+1} F_{j B}{ }_{n+1} F_{i A} C_{A B}^{t p,-1}
$$

$\tilde{\mathbf{b}}^{e}$ can be written in terms of ${ }_{n} \mathbf{b}^{e}$ by noting that the ${ }_{n} \mathbf{b}^{e}$ is defined by

$$
{ }_{n} b_{i j}^{e}={ }_{n} F_{j B}{ }_{n} F_{i A}{ }_{n} C_{A B}^{t p,-1}
$$

Comparing equations (73) and (74), we can write

$$
\tilde{b}_{i j}^{e}=f_{j l} f_{i k n} b_{k l}^{e}
$$

The incremental deformation gradient $\mathbf{f}$, which maps from the deformed configuration at time $\mathrm{n}$ and the deformed configuration at time $\mathrm{n}+1$, is defined as

$$
f_{i j}={ }_{n+1} F_{i A n} F_{A j}^{-1}
$$

To complete the update equation, taking the trace of (72) gives

$$
{ }_{n+1} b_{k k}^{e}=\theta \tilde{b}_{k k}^{e}
$$

which when plugged back into (72) and leads to

$$
{ }_{n+1} b_{i j}^{e}=\theta\left[\tilde{b}_{i j}^{e}-\frac{2}{3} \theta \Delta t_{n+1} g\left(\bar{\sigma}, T, \bar{\gamma}^{p}\right){ }_{n+1} n_{i j} \tilde{b}_{k k}^{e}\right]
$$

The update equation for $\mathbf{b}^{e}$ is thus defined in terms of $\tilde{\mathbf{b}}^{e}$, which depends on $\mathbf{f}$ and ${ }_{n} \mathbf{b}^{e}$, as well as the current iterates of $T, \boldsymbol{\sigma}$, and $\bar{\gamma}^{p}$.

In order to determine the pressure $p$ from equation (87), $J^{e}$ is needed. This can be computed in a few different ways, the simplest being

$$
J^{e}=\frac{1}{\aleph} \operatorname{det}(\mathbf{F})
$$

However, this requires knowledge of the original undeformed configuration for the computation of $\mathbf{F}$. If remeshing is needed, which is often the case for shear band modeling, it is desireable to use an updated Lagrangian framework where all quantities are expressed in terms of the deformed configurations at time 
steps $n$ and $n+1$. If this is the case, the elastic jacobian determinant could be updated as a history variable by numerically integrating the equation

$$
\begin{aligned}
\dot{J}^{e} & =J^{e} d_{k k}^{e} \\
& =J^{e}\left(d_{k k}-d_{k k}^{t}\right)
\end{aligned}
$$

Alternatively, $J^{e}$ could be computed by taking the determinant of equation (19) and solving for $J^{e}$

$$
J^{e}=\sqrt{\operatorname{det}\left(\mathbf{b}^{e}\right)}
$$

With these update equations for the elastic jacobian determinant, the entire formulation requires only quantities associated with time steps $n$ and $n+1$. Therefore, if the reference configuration is chosen to be the deformed configuration at time step n, then no quantities associated with the original, undeformed configuration are needed, allowing an updated Lagrangian approach to be used with remeshing if necessary. In the numerical results shown in this work, no remeshing is used, and therefore equation (79) is used.

\subsubsection{Neo Hookean Model}

The Neo Hookean energy potential is

$$
\phi^{N H}=U\left(J^{e}\right)+\frac{1}{2} \mu \bar{C}_{s s}^{e}
$$

Where $U$ is a function of the elastic Jacobian describing the volumetric contribution to the free energy and $\bar{C}_{s t}^{e}=J^{e,-2 / 3} C_{s t}^{e}$ is the isochoric part of the elastic right Cauchy Green tensor, since $\operatorname{det}\left(\overline{\mathbf{C}}^{e}\right)=1$. The volumetric contribution $U$ may be temperature dependent, to model a nonlinear relationship between temperature and pressure for materials undergoing shock compression. In this work, a temperature independent logarithmic form of $U$ is employed [Auricchio and Taylor, 1999]

$$
U=\frac{1}{2} K\left[\log \left(J^{e}\right)\right]^{2}
$$

Then from (53), the Kirchhoff stress is given by

$$
\tau_{i j}=K \log \left(J^{e}\right) \delta_{i j}+\mu \operatorname{dev}\left(\bar{b}_{i j}^{e}\right)
$$

where $K$ is the bulk modulus and

$$
\bar{b}_{i j}^{e}=J^{e,-2 / 3} b_{i j}^{e}
$$

This gives a volumetric - deviatoric split of the stress response, where the pressure $p$ is given by

$$
p=K \log \left(J^{e}\right)
$$


and the deviatoric part of the Kirchhoff stress is

$$
\operatorname{dev}\left(\tau_{i j}\right)=\mu \operatorname{dev}\left(\bar{b}_{i j}^{e}\right)
$$

\subsubsection{Logarithmic Based Hyperelastic Model}

The Hencky strain based hyperelastic model may be viewed as a generalization of the linear theory, where the elastic small strain tensor is replaced by the elastic Hencky strain.[Hencky, 1928] The elastic part of the free energy is thus given by

$$
\phi^{H S}=\frac{\lambda}{2} v_{k k}^{e, 2}+\mu v_{i j}^{e} v_{i j}^{e}
$$

Where $\mu$ is once again the shear modulus and $\lambda$ is Lame's constant. The Kirchhoff stress is then given by

$$
\tau_{i j}=\lambda v_{k k}^{e} \delta_{i j}+2 \mu v_{i j}^{e}
$$

\subsection{Grade zero hypoelastic formulation based on the Jaumann rate}

A hypoelastic formulation based on the Jaumann rate is used commonly for shear bands, see [Batra and Kim, 1992, Belytschko et al., 1994, BonnetLebouvier et al., 2002, Li et al., 2002, McAuliffe and Waisman, 2014, Nacar et al., 1989, Zhou et al., 1996] among others. This formulation can be obtained by placing two restricting assumptions on the rate form of the hyperelastic law (62), in addition to the constitutive assumptions made in the previous section. First, the spatial elastic moduli $c_{i j k l}^{\text {elas }}$ are assumed to be constant and isotropic so that

$$
c_{i j k l}^{\text {elas }}=c_{i j k l}^{\text {elas,iso }}=\lambda \delta_{i j} \delta_{k l}+\mu\left(\delta_{i k} \delta_{j l}+\delta_{i l} \delta_{j k}\right)
$$

Where $\lambda$ and $\mu$ are Lame's parameters. This is tantamount to the assumption that the elastic stretches are small so that the moduli $c_{i j k l}^{e l a s}$ remain constant. Such a material is often called a grade zero hypoelastic material and has been criticized for lack of general usefulness because it can be shown that such a material is incompatible with hyperelasticity, meaning that a hyperelastic potential such as $\phi$ cannot exist except when $\lambda=\mu$ [Simo and Pister, 1984]. In addition, such a material is not elastic in the sense that net work around a closed loop is non zero [Bernstein, 1960, Kojić and Bathe, 1987]. These issues are not unique to the Jaumann rate; a grade zero hypoelastic material based on any objective rate except one will not define an elastic material. The one exception is the logarithmic rate which was developed and analyzed in [Bruhns et al., 1999, Xiao et al., 1997a,b, 1999, 2000]. Those authors have demonstrated that the stress response of a grade zero hypoelastic material using the logarithmic rate is integrable to the response Hencky strain based hyperelastic model given by equation (90). Thus, the only possible differences 
between the two representations would be due to differences in the numerical techniques that were employed in each case. In this work, we only show results from total form. Further discussion and analysis of the logarithmic rate may be found in [Freed, 2014, Naghdabadi et al., 2005, Shutov and Ihlemann, 2014], and numerical simulations of elastic-plastic materials employing this rate may be found in [Brepols et al., 2014, Zhu et al., 2014].

For metal plasticity, the assumption of constant elastic moduli is generally appropriate since elastic stretches often remain small, however this may not be the case for high rate loading where large pressures and moderate volumetric deformations may be encountered. In the numerical results shown below, it is found that the maximum principal elastic stretches remain below 1.07 corresponding to a maximum logarithmic strain of about $6.7 \%$. Despite this, there are significant differences in the two formulations due to nonlinear elastic effects, as well as due to the spurious elastic energy dissipation caused by the hypoelastic model.

The second assumption that will be placed for the rate on the grade zero hypoelastic formulation is that the stress is negligible in comparison to the elastic moduli. This is the case for metals where the yield stress is often smaller than the elastic moduli by a few orders of magnitude. An identical assumption was placed on the hyperelastic formulation in the previous section, and as a consequence the form of the update equations for $\mathbf{b}^{e}$ were simplified. Similarly, with this assumption equation (62) can be written as

$$
\begin{aligned}
£_{v} \tau_{i j} & =c_{i j k l}^{\text {elas }, i s o} d_{k l}^{e}-\left(d_{i k}-d_{i k}^{e}\right) \tau_{k j}-\tau_{i k}\left(d_{j k}-d_{j k}^{e}\right) \\
& \approx c_{i j k l}^{\text {elas }, i s o} d_{k l}^{e}-d_{i k} \tau_{k j}-\tau_{i k} d_{j k}
\end{aligned}
$$

or

$$
\stackrel{\nabla}{\tau}_{i j}=c_{i j k l}^{\text {elas, } i s o} d_{k l}^{e}
$$

This is the Jaumann rate of Kirchhoff stress

$$
\stackrel{\nabla}{\tau}_{i j}=\dot{\tau}_{i j}-\omega_{i k} \tau_{k j}+\tau_{i k} \omega_{k j}=£_{v} \tau_{i j}+d_{i k} \tau_{k j}+\tau_{i k} d_{j k}
$$

The examination of various objective stress rates for grade zero hypoelastic and hypoelastic - plastic materials has been conducted in numerous studies [Atluri, 1984, Dienes, 1979, Fish and Shek, 2000, Flanagan and Taylor, 1987, Govindjee, 1997, Johnson and Bammann, 1984, Moss, 1984, Szabó and Balla, 1989, Zhou and Tamma, 2003]. Use of the grade zero hypoelastic material with Jaumann rate results in spurious oscillations in the stresses for large deformation simple shear, while other objective rates do not result in oscillations. The oscillations have also been resolved using a grade one hypoelastic material with non constant elastic moduli [Atluri, 1984], as well a corotational rate form hyperelastic formulation [Fish and Shek, 2000]. 
Despite the oscillations, under conditions where elastic strains are indeed small and cyclic loading is not encountered, the grade zero hypoelastic plastic material with the Jaumann rate matches well with experiments [Weber and Anand, 1990]. In addition, this formulation has the significant numerical advantage that a symmetric tangent modulus can be obtained [Simo and Hughes, 1998], permitting the use of a symmetric linear solver when implicit dynamics are used. However, note that not all objective rates lead to symmetric moduli. Regardless of whether or not the specific rate chosen leads to symmetric moduli, this advantage is negated when the global monolithic shear band solver is employed, where a non symmetric linear solver must be used for both the hyper and hypoelastic formulations. This is because the problem involves nonsymmetric thermo mechanical coupling. This is likely to also be the case for most monolithic solvers for multiphysics problems involving plasticity. In light of this, the only advantage the grade zero hypoelastic formulation for shear band modeling is that elastic deformation measures such as $J^{e}$ and $\mathbf{b}^{e}$ do not have to be stored as history variables, though this only leads to minor memory savings.

Table 1 summarizes the time discrete forms of the implicit equations for stress for each model. Neither discrete model stands out as significantly simpler than the other in terms of ease of implementation in the global monolithic solution framework.

In the numerical results below, we only show results for the Jaumann rate, and do not examine several objective rates. There are two reasons for this, the first being that the Jaumann rate is the rate that is most often used for shear band modeling. The second reason is that with the exception of the logarithmic rate, the deficiencies of the grade zero hypoelastic formulation will not be resolved by replacing the Jaumann rate with some other objective rate. While the logarithmic rate would certainly fix these deficiencies, it is equivalent to the Hencky strain based hyperelastic model, and is therefore also not shown.

\section{Numerical results}

The two formulations described above have been implemented in the monolithic solution framework described in [McAuliffe and Waisman, 2013, 2014]. Computations were done in the finite element code FEAP version 8.3 [Taylor, 2011], and the results were post processed using Paraview [Henderson, 2012] and Matplotlib [Hunter, 2007]. PETSc version 3.4 [Balay et al., 1997, 2014a,b] was used to assemble and solve the global equation system where the sparse direct linear solver UMFPACK [Davis, 2004] was the particular solver chosen. The material parameters were chosen as representative of structural steel, see table 2. The flow law $g\left(\bar{\sigma}, T, \bar{\gamma}^{p}\right)$ is phenomenological [Zhou et al., 1996], and 
Table 1

Summary of the implicit equations for stress for the hyper and hypoelastic formulations, discretized in time with backward Euler. Quantities used for both formulations are in the top section of the table. Quantities are taken at time step $n+1$ unless explicitly noted otherwise.

\begin{tabular}{|l|l|}
\hline Quantitities used by all models & $f_{i j}=F_{i A}{ }_{n} F_{A j}^{-1}$ \\
& $d_{i j}^{p}=\frac{3}{2} g\left(\bar{\sigma}, T, \bar{\gamma}^{p}\right) \frac{s_{i j}}{\bar{\sigma}}$ \\
& $d_{i j}^{t}=\alpha \dot{T} \delta_{i j}$ \\
\hline \multirow{4}{*}{ Hyperelastic, Neo Hookean } & $\tau_{i j}^{N H}=K \log \left(J^{e}\right) \delta_{i j}+\mu \operatorname{dev}\left(\bar{b}_{i j}^{e}\right)$ \\
& $J^{e}=\frac{1}{\aleph} \operatorname{det}(\mathbf{F})$ \\
& $b_{i j}^{e}=\theta\left[\tilde{b}_{i j}^{e}-\frac{2}{3} \theta \Delta t{ }_{n+1} d_{i j}^{p} \tilde{b}_{k k}^{e}\right]$ \\
& $\tilde{b}_{i j}^{e}=f_{i k} f_{j l}{ }_{n} b_{k l}^{e}$ \\
& $\theta=\left(1+2 \Delta t \alpha{ }_{n+1} \dot{T}\right)^{-1}$ \\
\hline Hyperelastic, Hencky strain model & $\tau_{i j}^{H S}=\lambda v_{k k}^{e} \delta_{i j}+2 \mu v_{i j}^{e}$ \\
& $J^{e}=\frac{1}{\aleph} \operatorname{det}(\mathbf{F})$ \\
& $b_{i j}^{e}=\theta\left[\tilde{b}_{i j}^{e}-\frac{2}{3} \theta \Delta t{ }_{n+1} d_{i j}^{p} \tilde{b}_{k k}^{e}\right]$ \\
& $\tilde{b}_{i j}^{e}=f_{i k} f_{j l}{ }_{n} b_{k l}^{e}$ \\
& $\theta=\left(1+2 \Delta t \alpha{ }_{n+1} \dot{T}\right)^{-1}$ \\
\hline Hypoelastic, Jaumann rate & $\tau_{i j}=\tilde{\tau}_{i j}+c_{i j k l}^{e l a s, i s o} d_{k l}^{e}-d_{i k} \tau_{k j}-\tau_{i k} d_{k j}$ \\
& $\tilde{\tau}_{i j}=f_{i k} f_{j l}{ }_{n} \tau_{k l}$ \\
& $c_{i j k l}^{e l a s, i s o}=\lambda \delta_{i j} \delta_{k l}+\mu\left(\delta_{i k} \delta_{j l}+\delta_{i l} \delta_{j k}\right)$ \\
& $d_{i j}^{e}=d_{i j}-d_{i j}^{p}-d_{i j}^{t}$ \\
& $\Delta t d_{i j}=\frac{1}{2}\left[\delta_{i j}-f_{k i}^{-1} f_{k j}^{-1}\right]$ \\
\hline
\end{tabular}

employs power law strain and strain rate hardening, and exponential thermal softening as follows

$$
g\left(\bar{\sigma}, T, \bar{\gamma}^{p}\right)=\dot{\gamma}_{0}\left[\frac{\bar{\sigma}}{\sigma_{0}\left(1+\bar{\gamma}^{p} / \gamma_{0}\right)^{n}\left\{1-\delta\left[\exp \left(\frac{T-T_{0}}{k}\right)-1\right]\right\}}\right]^{m}
$$

Phenomenological models with qualitatively similar behavior may be found in [Bodner and Partom, 1975, Johnson and Cook, 1985, Khan and Huang, 1992, Khan and Liang, 1999, Zerilli and Armstrong, 1987]. 
Table 2

Material Properties and Parameters

\begin{tabular}{|c|c|c|c|}
\hline Property Name & Symbol & Value & Unit \\
\hline \hline Young's Modulus & $\mathrm{E}$ & $200 \mathrm{E} 9$ & $\mathrm{~Pa}$ \\
\hline Poisson's Ratio & $\nu$ & 0.3 & - \\
\hline Mass Density & $\rho$ & 7830 & $\frac{\mathrm{kg}}{\mathrm{m}^{3}}$ \\
\hline Specific Heat & $\mathrm{c}$ & 448 & $\frac{\mathrm{J}}{\mathrm{kgK}}$ \\
\hline Taylor - Quinney Coefficient & $\chi$ & 0.9 & - \\
\hline Thermal Conductivity & $\kappa$ & 50.0 & $\frac{\mathrm{W}}{\mathrm{mK}}$ \\
\hline Thermal Expansion Coefficient & $\alpha$ & $11.0 \mathrm{E}-6$ & $\frac{1}{K}$ \\
\hline Reference Strain Rate & $\dot{\gamma}_{0}$ & 0.001 & $\frac{1}{s}$ \\
\hline Rate Sensitivity Parameter & $\mathrm{m}$ & 70 & - \\
\hline Yield Stress & $\sigma_{0}$ & $2000 \mathrm{E} 6$ & $\mathrm{~Pa}$ \\
\hline Yield Strain & $\gamma_{0}$ & 0.01 & - \\
\hline Strain Hardening Exponent & $\mathrm{n}$ & 0.01 & - \\
\hline Reference Temperature & $T_{0}$ & 293 & $\mathrm{~K}$ \\
\hline Thermal Softening Parameter & $\delta$ & 0.8 & - \\
\hline Thermal Softening Parameter & $\mathrm{k}$ & 500 & $\mathrm{~K}$ \\
\hline
\end{tabular}

\subsection{Plate in Tension}

The first problem studied is a plate under plane strain tension, illustrated in Figure 2. A velocity of $2 \mathrm{~m} / \mathrm{s}$ is applied to the top edge, and a smooth imperfection is applied to the model such that the initial yield stress is lowest at the lower left corner of the modeled domain. This will cause a shear band to form from this corner at about $45^{\circ}$.

Figure 3 shows the equivalent plastic strain distribution at the end of the simulation, where the hyperelastic formulation leads to higher strains and more intense localization. On the other hand, examination of the equivalent plastic strain and Von Mises stress vs time in Figure 4, shows that the two formulations are indistinguishable up until the Von Mises stress has dropped to about $85 \%$ of its maximum value.

For the hyperelastic case, the elastic moduli start initially at the ground state values and decrease as the elastic strain increases. Thus, the hyper and hypoelastic formulations are nearly identical early on, but depart significantly after softening where the reduced elastic moduli of the hyperelastic formulation lead 
to stronger localization and more intense inelastic straining. Since the moduli are deformation dependent, the deformation process leads to heterogeneity in the elastic moduli throughout the material. For a given input nominal strain, the more compliant areas of the material will have higher local strains than the more compliant areas. Thus hyperelasticity effects shear band formation locally, where the reduced moduli within the shear band leads to more intense straining, as well as globally, due to the heterogeneous distribution of the elastic moduli leading to a higher proportion of the strain input to concentrate in the already heavily deformed shear band.

There are not noticeable differences between the two hyperelastic models until very late in the deformation, where the Hencky strain based model begins to localize more severely. Compared with a Neo Hookean model, the Hencky strain model will become more compliant with increasing deformation in shear and tension[Batra, 2001] (note however that the models used in this reference are slight variants of models used here), enhancing the effect described above. Since either model can be used to model finite elasticity in metals, we show results for the Neo Hookean model only for the remainder of the paper.

Table 3

Summary of computational effort for the plate in tension

\begin{tabular}{|c|c|c|c|c|}
\hline & Time Steps & Newton Iterations & CPU time (sec) & Max Memory Usage (bytes) \\
\hline \hline Hyperelastic, NH & 130 & 507 & 286.33 & $1.24314 \mathrm{E}+08$ \\
\hline Hyperelastic, HS & 130 & 510 & 333.25 & $1.41242 \mathrm{E}+08$ \\
\hline Hypoelastic & 130 & 446 & 253.7 & $1.41668 \mathrm{E}+08$ \\
\hline
\end{tabular}

From a numerical perspective, the two formulations are comparable, as summarized in table 3. The hyperelastic formulation requires more Newton iterations due to the extra source of nonlinearity from the hyperelastic law and the more intense localization that results from it.

\subsection{Plate in Compression}

The next problem studied is a plate subject to compression, as shown in Figure 5 A velocity of $25 \mathrm{~m} / \mathrm{s}$ is applied downward on the top edge. Due to the geometry of the problem, no imperfection in material properties is needed to nucleate a shear band. This problem setup results in shearing with a compressive component.

Figure 10 shows surface plots of the equivalent plastic strain on the deformed mesh. For both formulations, a well developed shear band is present, but the intensity of the shear band is much higher for the hyperelastic formulation. This is illustrated further in Figure 7, where the equivalent plastic strain and 
temperature profiles are plotted across the shear band. The peak temperature is nearly identical for both formulations, since the heat produced by shearing is related to both the stress and the plastic strain rate. In comparison to the hypoelastic formulation, the hyperelastic formulation leads to decreased stress but increased plastic strain rate, resulting in similar rates of heat production.

To show the global behavior, the mechanical work is examined. The domain integrated stress power $\dot{\mathcal{W}}$ is defined by

$$
\dot{\mathcal{W}}=\int_{V} \sigma_{i j} d_{i j} d V
$$

where $V$ is the deformed volume. This can be split with the additive decomposition of $\mathbf{d}$ into elastic, inelastic and thermal contributions as follows

$$
\begin{aligned}
\dot{\mathcal{W}}^{e} & =\int_{V} \sigma_{i j} d_{i j}^{e} d V \\
\dot{\mathcal{W}}^{p} & =\int_{V} \sigma_{i j} d_{i j}^{p} d V \\
\dot{\mathcal{W}}^{t} & =\int_{V} \sigma_{i j} d_{i j}^{t} d V
\end{aligned}
$$

Integrating the power in time gives the total mechanical work $\mathcal{W}$. Figure 8 shows the total, elastic, and inelastic contributions to the mechanical work. The thermal contribution is small and thus not shown. To make it easier to visualize the differences in the results, the ratios of these work quantities for the two formulations are plotted over the time interval of 2.0-5.0e-7 seconds in Figure 9 . The figures demonstrate that after roughly $3.5 \mathrm{e}-7$ seconds, the elastic contribution of the mechanical work begins to decrease more quickly for the hyperelastic formulation. Initially, this deviation in the elastic contribution is small and is offset by a slight increase in the inelastic contribution. Shortly thereafter however, the total mechanical work of the two formulations begin to depart. Since in both cases the work put into the system by the boundary conditions is the same, the decrease in mechanical work for the hyperelastic case requires a commensurate increase in the kinetic energy, which enhances localization.

For applications where energy absorption is of interest, the grade zero hypoelastic formulation will tend to overestimate the dissipation of the material, since nonlinear elastic effect clearly reduces energy absorption capability.

This problem is significantly more demanding than the smaller plate in tension problem, but, as summarized in table 4, the computational resources required for the two formulations are comparable, with about $9 \%$ more cpu time and $2 \%$ more memory for the hyperelastic model. 
Table 4

Summary of computational effort for the plate in compression

\begin{tabular}{|c|c|c|c|c|}
\hline & Time Steps & Newton Iterations & CPU time (sec) & Max Memory Usage (bytes) \\
\hline \hline Hyperelastic & 442 & 1816 & 5171.07 & $5.40967 \mathrm{E}+08$ \\
\hline Hypoelastic & 379 & 1673 & 4630.1 & $5.22576 \mathrm{E}+08$ \\
\hline
\end{tabular}

\subsection{Kalthoff Experiment}

Experiments on the impact of notched plates have demonstrated the well known ductile - brittle failure transition for metals and other materials. Here, a notched plat is loaded with three different impact velocities: 10, 20, and $30 \mathrm{~m} / \mathrm{s}$. Shear bands initiate for the two higher velocities while for $10 \mathrm{~m} / \mathrm{s}$ it does not lead to shear band formation. The geometry of the simulated problem can be found in [Zhou et al., 1996]. The equivalent plastic strain histories at the shear band initiation point are shown for the three impact velocities and the two formulations in Figure 11. Again, the formulations for 20 and $30 \mathrm{~m} / \mathrm{s}$ are very similar for small elastic strains, but depart rapidly as the strains grow, while for $10 \mathrm{~m} / \mathrm{s}$ the formulations are indistinguishable throughout the run. Previous studies on the ductile brittle transition have concluded that the formation and propagation of a shear band shields the material from developing the stresses needed for crack propagation. Therefore, this difference in behavior for the higher impact velocities is of interest for the failure transition because the timing of shear band formation determines whether or not the shielding effect occurs.

The differences between the two formulations become more pronounced as the strain rates increase. This is due to the rate dependence of plasticity, where higher strain rates delay the onset of plasticity, leading to higher elastic strains and a greater reduction in the elastic moduli.

Hyperelastic effects have also been shown to play a role in the dynamics of fracture in atomistic simulations [Abraham, 1996, Abraham et al., 1997a,b, Buehler and Gao, 2006, Buehler et al., 2003] as well as analysis on the continuum level [Gao, 1996]. These works have shown that the hyperelastic softening or stiffening due to large local elastic deformations in the vicinity of the crack tip lead to changes in the crack propagation speeds and the energy flow to the crack tip. Fracture is not explicitly modeled in this work, although the maximum principal stress at the top of the notch tip can be examined as a measure of the tendency of the material to develop a crack [Needleman and Tvergaard, 1995]. This is shown in Figure 11, where the largest principal stress attained by the hyperelastic formulation is smaller than the largest principal stress attained by the hyperelastic formulation. The largest principal elastic stretch in the same region does not exceed 1.015, and therefore we can surmise 
that the smaller principal stresses observed for the hyperelastic formulation are not the direct result of hyperelastic effects at the top of the notch tip, but rather are the result of the more rapid shear band development enhancing the shielding effect described by [Needleman and Tvergaard, 1995].

\section{Conclusions}

The nonlinear elastic softening effect of a neo Hookean hyperelastic model was found to significantly enhance localization in shear band modeling by reducing the compliance of the material. This occurred even though the elastic strains developed in all of the simulations reported could not be considered large. Evidently, relatively small reductions in the elastic moduli lead to significant differences in the intensity of inelastic straining, but only once the material is in the highly nonlinear localization regime. The nonlinear elastic effects were shown to predict lower energy dissipation than the grade zero hypoelastic formulation that is typically used for shear bands. The role of the nonlinear elastic effect is rate dependent, since the rate dependence of plasticity delays yielding for higher strain rates, which leads to larger elastic strains and greater reduction of the elastic moduli. This is of interest in the ductile - brittle failure transition, which is governed by the competition between the formation of shear bands due to thermal softening, and the development of a crack due to large principal stress [Needleman and Tvergaard, 1995, 2000]. Hyperelasticity will affect both aspects of the transition since as shown here, the nonlinear elastic softening accelerates the formation of a shear band, and as shown by [Buehler et al., 2003], nonlinear elastic softening decrease the energy flow to the crack tip. Thus, in comparison to a linear elastic solid, elastic softening material will shear band more readily and fracture less readily. For a material that hardens elastically, the opposite behavior is expected. 


\section{Acknowledgment}

The financial support of the U.S Department of Energy office of Science, through the Early Career Research Program, No. DE-SC-0008196, and the Army Research Office, under grant No. W911NF1310238, is gratefully acknowledged.

\section{References}

Farid F. Abraham. Dynamics of brittle fracture with variable elasticity. Physical Review Letters, 77(5):869-872, July 1996. doi: 10.1103/PhysRevLett.77.869.

Farid F. Abraham, Dominique Brodbeck, William E. Rudge, and Xiaopeng Xu. A molecular dynamics investigation of rapid fracture mechanics. Journal of the Mechanics and Physics of Solids, 45(9):1595-1619, September 1997a. ISSN 0022-5096. doi: 10.1016/S0022-5096(96)00103-2.

Farid F. Abraham, D. Schneider, B. Land, D. Lifka, J. Skovira, J. Gerner, and M. Rosenkrantz. Instability dynamics in three-dimensional fracture: An atomistic simulation. Journal of the Mechanics and Physics of Solids, 45(9):1461-1471, September 1997b. ISSN 0022-5096. doi: 10.1016/S00225096(97)00017-3.

L. Anand. On h. hencky's approximate strain-energy function for moderate deformations. Journal of Applied Mechanics, 46(1):78-82, March 1979. ISSN 0021-8936. doi: 10.1115/1.3424532.

L. Anand, M. E. Gurtin, S. P. Lele, and C. Gething. A one-dimensional theory of strain-gradient plasticity: Formulation, analysis, numerical results. Journal of the Mechanics and Physics of Solids, 53(8):1789-1826, August 2005. ISSN 0022-5096. doi: 10.1016/j.jmps.2005.03.003.

Lallit Anand, Ozgur Aslan, and Shawn A. Chester. A large-deformation gradient theory for elastic-plastic materials: Strain softening and regularization of shear bands. International Journal of Plasticity, 30-31:116-143, March 2012. ISSN 0749-6419. doi: 10.1016/j.ijplas.2011.10.002.

José E. Andrade and Ronaldo I. Borja. Capturing strain localization in dense sands with random density. International Journal for Numerical Methods in Engineering, 67(11):1531-1564, September 2006. ISSN 1097-0207. doi: 10.1002/nme.1673.

Miguel Arriaga, Colin McAuliffe, and Haim Waisman. Onset of shear band localization by a local generalized eigenvalue analysis. Computer Methods in Applied Mechanics and Engineering, 289:179-208, June 2015. ISSN 00457825. doi: 10.1016/j.cma.2015.02.010. 
Satya N. Atluri. On constitutive relations at finite strain: Hypo-elasticity and elasto-plasticity with isotropic or kinematic hardening. Computer Methods in Applied Mechanics and Engineering, 43(2):137-171, April 1984. ISSN 0045-7825. doi: 10.1016/0045-7825(84)90002-1.

F. Auricchio and R.L. Taylor. A return-map algorithm for general associative isotropic elasto-plastic materials in large deformation regimes. International Journal of Plasticity, 15(12):1359-1378, January 1999. ISSN 07496419. doi: 10.1016/S0749-6419(99)00044-3.

Y. L. Bai. Thermo-plastic instability in simple shear. Journal of the Mechanics and Physics of Solids, 30(4):195-207, August 1982. ISSN 0022-5096. doi: 10.1016/0022-5096(82)90029-1.

Satish Balay, William D. Gropp, Lois Curfman McInnes, and Barry F. Smith. Efficient management of parallelism in object oriented numerical software libraries. In E. Arge, A. M. Bruaset, and H. P. Langtangen, editors, Modern Software Tools in Scientific Computing, pages 163-202. Birkh"auser Press, 1997.

Satish Balay, Shrirang Abhyankar, Mark F. Adams, Jed Brown, Peter Brune, Kris Buschelman, Victor Eijkhout, William D. Gropp, Dinesh Kaushik, Matthew G. Knepley, Lois Curfman McInnes, Karl Rupp, Barry F. Smith, and Hong Zhang. PETSc users manual. Technical Report ANL-95/11 Revision 3.5, Argonne National Laboratory, 2014a.

Satish Balay, Shrirang Abhyankar, Mark F. Adams, Jed Brown, Peter Brune, Kris Buschelman, Victor Eijkhout, William D. Gropp, Dinesh Kaushik, Matthew G. Knepley, Lois Curfman McInnes, Karl Rupp, Barry F. Smith, and Hong Zhang. PETSc web page. 2014b.

R. C Batra. Comparison of results from four linear constitutive relations in isotropic finite elasticity. International Journal of Non-Linear Mechanics, 36 (3):421-432, May 2001. ISSN 0020-7462. doi: 10.1016/S0020-7462(00)000573 .

R. C. Batra and C. H. Kim. Analysis of shear banding in twelve materials. International Journal of Plasticity, 8(4):425-452, 1992. ISSN 0749-6419. doi: 10.1016/0749-6419(92)90058-K.

Ted Belytschko, Huai-Yang Chiang, and Edward Plaskacz. High resolution two-dimensional shear band computations: imperfections and mesh dependence. Computer Methods in Applied Mechanics and Engineering, 119(1-2): 1-15, November 1994. ISSN 0045-7825. doi: 10.1016/0045-7825(94)00073-5.

Barry Bernstein. Hypo-elasticity and elasticity. Archive for Rational Mechanics and Analysis, 6(1):89-104, January 1960. ISSN 0003-9527, 1432-0673. doi: $10.1007 / \mathrm{BF} 00276156$.

S. R. Bodner and Y. Partom. Constitutive equations for elastic-viscoplastic strain-hardening materials. Journal of Applied Mechanics, 42(2):385-389, June 1975. ISSN 0021-8936. doi: 10.1115/1.3423586.

A.-S. Bonnet-Lebouvier, A. Molinari, and P. Lipinski. Analysis of the dynamic propagation of adiabatic shear bands. International Journal of Solids and Structures, 39(16):4249-4269, 2002. 
Ronaldo I. Borja. Bifurcation of elastoplastic solids to shear band mode at finite strain. Computer Methods in Applied Mechanics and Engineering, 191 (46):5287-5314, 2002.

Ronaldo I. Borja, Xiaoyu Song, Amy L. Rechenmacher, Sara Abedi, and Wei $\mathrm{Wu}$. Shear band in sand with spatially varying density. Journal of the Mechanics and Physics of Solids, 61(1):219-234, January 2013. ISSN 00225096. doi: 10.1016/j.jmps.2012.07.008.

Tim Brepols, Ivaylo N. Vladimirov, and Stefanie Reese. Numerical comparison of isotropic hypo- and hyperelastic-based plasticity models with application to industrial forming processes. International Journal of Plasticity, 63:1848, December 2014. ISSN 0749-6419. doi: 10.1016/j.ijplas.2014.06.003.

O. T. Bruhns, H. Xiao, and A. Meyers. Self-consistent eulerian rate type elasto-plasticity models based upon the logarithmic stress rate. International Journal of Plasticity, 15(5):479-520, April 1999. ISSN 0749-6419. doi: 10.1016/S0749-6419(99)00003-0.

Markus J. Buehler and Huajian Gao. Dynamical fracture instabilities due to local hyperelasticity at crack tips. Nature, 439(7074):307-310, January 2006. ISSN 0028-0836. doi: 10.1038/nature04408.

Markus J. Buehler, Farid F. Abraham, and Huajian Gao. Hyperelasticity governs dynamic fracture at a critical length scale. Nature, 426(6963):141146, 2003.

Yannis F. Dafalias. Plastic spin: necessity or redundancy? International Journal of Plasticity, 14(9):909-931, December 1998. ISSN 0749-6419. doi: 10.1016/S0749-6419(98)00036-9.

Timothy A. Davis. Algorithm 832: UMFPACK v4.3 - an unsymmetric-pattern multifrontal method. ACM Trans. Math. Softw., 30(2):196-199, June 2004. ISSN 0098-3500. doi: 10.1145/992200.992206.

J. K. Dienes. On the analysis of rotation and stress rate in deforming bodies. Acta Mechanica, 32(4):217-232, December 1979. ISSN 0001-5970, 16196937. doi: 10.1007/BF01379008.

Adrian Luis Eterovic and Klaus-Jürgen Bathe. A hyperelastic-based large strain elasto-plastic constitutive formulation with combined isotropickinematic hardening using the logarithmic stress and strain measures. International Journal for Numerical Methods in Engineering, 30(6):1099-1114, 1990.

Jacob Fish and Kamlun Shek. Finite deformation plasticity based on the additive split of the rate of deformation and hyperelasticity. Computer Methods in Applied Mechanics and Engineering, 190(1-2):75-93, October 2000. ISSN 0045-7825. doi: 10.1016/S0045-7825(99)00415-6.

D. P. Flanagan and L. M. Taylor. An accurate numerical algorithm for stress integration with finite rotations. Computer Methods in Applied Mechanics and Engineering, 62(3):305-320, June 1987. ISSN 0045-7825. doi: 10.1016/0045-7825(87)90065-X.

A. D. Freed. Hencky strain and logarithmic rates in lagrangian analysis. International Journal of Engineering Science, 81:135-145, August 2014. ISSN 
0020-7225. doi: 10.1016/j.ijengsci.2014.04.016.

C. Fressengeas and A. Molinari. Instability and localization of plastic flow in shear at high strain rates. Journal of the Mechanics and Physics of Solids, 35(2):185-211, 1987. ISSN 0022-5096. doi: 10.1016/0022-5096(87)90035-4.

Huajian Gao. A theory of local limiting speed in dynamic fracture. Journal of the Mechanics and Physics of Solids, 44(9):1453-1474, September 1996. ISSN 0022-5096. doi: 10.1016/0022-5096(96)00038-5.

Sanjay Govindjee. Accuracy and stability for integration of jaumann stress rate equations in spinning bodies. Engineering Computations, 14(1):14-30, 1997. ISSN 0264-4401. doi: 10.1108/02644409710157604.

Morton E. Gurtin and Lallit Anand. A theory of strain-gradient plasticity for isotropic, plastically irrotational materials. part II: Finite deformations. International Journal of Plasticity, 21(12):2297-2318, December 2005. ISSN 0749-6419. doi: 10.1016/j.ijplas.2005.01.006.

H. Hencky. Über die Form des Elastizitätsgesetzes bei ideal elastischen Stoffen. Z. Techn. Phys., 9:215-220, 1928.

A. Henderson. ParaView Guide, A Parallel Visualization Application, Kitware Inc. 4 edition, 2012. ISBN 978-1-1930934-24-5.

J.D. Hunter. Matplotlib: A 2d graphics environment. Computing in Science Engineering, 9(3):90-95, May 2007. ISSN 1521-9615. doi: 10.1109/MCSE.2007.55.

Alexander V. Idesman. Comparison of different isotropic elastoplastic models at finite strains used in numerical analysis. Computer Methods in Applied Mechanics and Engineering, 192(41-42):4659-4674, October 2003. ISSN 0045-7825. doi: 10.1016/S0045-7825(03)00444-4.

George C. Johnson and Douglas J. Bammann. A discussion of stress rates in finite deformation problems. International Journal of Solids and Structures, 20(8):725-737, 1984. ISSN 0020-7683. doi: 10.1016/0020-7683(84)90061-1.

Gordon R. Johnson and William H. Cook. Fracture characteristics of three metals subjected to various strains, strain rates, temperatures and pressures. Engineering Fracture Mechanics, 21(1):31-48, 1985. ISSN 0013-7944. doi: 10.1016/0013-7944(85)90052-9.

Akhtar S. Khan and Sujian Huang. Experimental and theoretical study of mechanical behavior of 1100 aluminum in the strain rate range 10-5-104s-1. International Journal of Plasticity, 8(4):397-424, 1992. ISSN 0749-6419. doi: 10.1016/0749-6419(92)90057-J.

Akhtar S. Khan and Riqiang Liang. Behaviors of three BCC metal over a wide range of strain rates and temperatures: experiments and modeling. International Journal of Plasticity, 15(10):1089-1109, 1999. ISSN 07496419. doi: 10.1016/S0749-6419(99)00030-3.

Miloš Kojić and Klaus-Jürgen Bathe. Studies of finite element procedures - stress solution of a closed elastic strain path with stretching and shearing using the updated lagrangian jaumann formulation. Computers Ef Structures, 26(1-2):175-179, 1987. ISSN 0045-7949. doi: 10.1016/00457949(87)90247-1. 
Yves M. Leroy and Alain Molinari. Spatial patterns and size effects in shear zones : A hyperelastic model with higher-order gradients. Journal of the Mechanics and Physics of Solids, 41(4):631-663, April 1993. ISSN 00225096. doi: 10.1016/0022-5096(93)90021-7.

Shaofan Li, Wing Kam Liu, Ares J. Rosakis, Ted Belytschko, and Wei Hao. Mesh-free galerkin simulations of dynamic shear band propagation and failure mode transition. International Journal of Solids and Structures, 39(5):1213-1240, March 2002. ISSN 0020-7683. doi: 10.1016/S00207683(01)00188-3.

Xianwu Ling and T. Belytschko. Thermal softening induced plastic instability in rate-dependent materials. Journal of the Mechanics and Physics of Solids, 57(4):788-802, April 2009. ISSN 00225096. doi: 10.1016/j.jmps.2008.04.010.

Vlado A Lubarda. Constitutive theories based on the multiplicative decomposition of deformation gradient: Thermoelasticity, elastoplasticity, and biomechanics. Applied Mechanics Reviews, 57(2):95, 2004. ISSN 00036900. doi: 10.1115/1.1591000.

Colin McAuliffe and Haim Waisman. Mesh insensitive formulation for initiation and growth of shear bands using mixed finite elements. Computational Mechanics, 51(5):807-823, May 2013. ISSN 0178-7675, 1432-0924. doi: $10.1007 / \mathrm{s} 00466-012-0765-\mathrm{z}$.

Colin McAuliffe and Haim Waisman. A pian-sumihara type element for modeling shear bands at finite deformation. Computational Mechanics, 53(5): 925-940, May 2014. ISSN 0178-7675, 1432-0924. doi: 10.1007/s00466-0130940-x.

Christian Miehe. On the representation of prandtl-reuss tensors within the framework of multiplicative elastoplasticity. International Journal of Plasticity, 10(6):609-621, 1994. ISSN 0749-6419. doi: 10.1016/07496419(94)90025-6.

William C. Moss. On instabilities in large deformation simple shear loading. Computer Methods in Applied Mechanics and Engineering, 46(3):329-338, November 1984. ISSN 0045-7825. doi: 10.1016/0045-7825(84)90108-7.

A. Nacar, A. Needleman, and M. Ortiz. A finite element method for analyzing localization in rate dependent solids at finite strains. Computer methods in applied mechanics and engineering, 73(3):235-258, 1989.

R. Naghdabadi, M. Yeganeh, and A. R. Saidi. Application of corotational rates of the logarithmic strain in constitutive modeling of hardening materials at finite deformations. International Journal of Plasticity, 21(8):1546-1567, August 2005. ISSN 0749-6419. doi: 10.1016/j.ijplas.2004.07.005.

A. Needleman and V. Tvergaard. Analysis of a brittle-ductile transition under dynamic shear loading. International Journal of Solids and Structures, 32 (17-18):2571-2590, September 1995. ISSN 0020-7683. doi: 10.1016/00207683(94)00283-3.

A. Needleman and V. Tvergaard. Numerical modeling of the ductile-brittle transition. International Journal of Fracture, 101(1-2):73-97, January 2000. ISSN 0376-9429, 1573-2673. doi: 10.1023/A:1007520917244. 
W. Prager. An elementary discussion of definitions of stress rates. Quarterly Applied Math, 18:403-407, 1961.

A. V. Shutov and J. Ihlemann. Analysis of some basic approaches to finite strain elasto-plasticity in view of reference change. International Journal of Plasticity, 63:183-197, December 2014. ISSN 0749-6419. doi: 10.1016/j.ijplas.2014.07.004.

J. C. Simo. A framework for finite strain elastoplasticity based on maximum plastic dissipation and the multiplicative decomposition: Part i. continuum formulation. Computer Methods in Applied Mechanics and Engineering, 66(2):199-219, February 1988. ISSN 0045-7825. doi: 10.1016/00457825(88)90076-X.

J. C Simo and Thomas J. R Hughes. Computational inelasticity. Springer, New York, 1998. ISBN 03872276369780387227634.

J. C. Simo and Ch Miehe. Associative coupled thermoplasticity at finite strains: formulation, numerical analysis and implementation. Computer Methods in Applied Mechanics and Engineering, 98(1):41-104, 1992.

J.C. Simo and K.S. Pister. Remarks on rate constitutive equations for finite deformation problems: computational implications. Computer Methods in Applied Mechanics and Engineering, 46(2):201-215, October 1984. ISSN 00457825. doi: 10.1016/0045-7825(84)90062-8.

László Szabó and Mihály Balla. Comparison of some stress rates. International Journal of Solids and Structures, 25(3):279-297, 1989. ISSN 0020-7683. doi: 10.1016/0020-7683(89)90049-8.

Robert L. Taylor. FEAP-A Finite Element Analysis Program. Univ. of Calif., Berkeley, 2011.

N. Triantafyllidis and Elias C. Aifantis. A gradient approach to localization of deformation. i. hyperelastic materials. Journal of Elasticity, 16(3):225-237, September 1986. ISSN 0374-3535, 1573-2681. doi: 10.1007/BF00040814.

Gustavo Weber and Lallit Anand. Finite deformation constitutive equations and a time integration procedure for isotropic, hyperelastic-viscoplastic solids. Computer Methods in Applied Mechanics and Engineering, 79(2): 173-202, March 1990. ISSN 0045-7825. doi: 10.1016/0045-7825(90)90131-5.

T. W. Wright. The physics and mathematics of adiabatic shear bands. Cambridge monographs on mechanics. Cambridge University Press, Cambridge, UK ; New York, 2002. ISBN 0521631955.

H. Xiao, O. T. Bruhns, and A. Meyers. Logarithmic strain, logarithmic spin and logarithmic rate. Acta Mechanica, 124(1-4):89-105, March 1997a. ISSN 0001-5970, 1619-6937. doi: 10.1007/BF01213020.

H. Xiao, O. T. Bruhns, and A. Meyers. Hypo-elasticity model based upon the logarithmic stress rate. Journal of Elasticity, 47(1):51-68, April 1997b. ISSN 0374-3535, 1573-2681. doi: 10.1023/A:1007356925912.

H. Xiao, O. T. Bruhns, and A. Meyers. Existence and uniqueness of the integrable-exactly hypoelastic equation $\stackrel{\circ}{\tau}=\lambda(\operatorname{tr} D) I+2 \mu D$ and its significance to finite inelasticity. Acta Mechanica, 138(1-2):31-50, March 1999. ISSN 0001-5970, 1619-6937. doi: 10.1007/BF01179540. 
H. Xiao, O. T. Bruhns, and A. Meyers. The choice of objective rates in finite elastoplasticity: general results on the uniqueness of the logarithmic rate. Proceedings of the Royal Society of London. Series A: Mathematical, Physical and Engineering Sciences, 456(2000):1865-1882, August 2000. ISSN 1364-5021, 1471-2946. doi: 10.1098/rspa.2000.0591.

Frank J. Zerilli and Ronald W. Armstrong. Dislocation-mechanics-based constitutive relations for material dynamics calculations. Journal of Applied Physics, 61(5):1816-1825, March 1987. ISSN 0021-8979, 1089-7550. doi: $10.1063 / 1.338024$.

M. Zhou, G. Ravichandran, and A. J. Rosakis. Dynamically propagating shear bands in impact-loaded prenotched plates - II. numerical simulations. Journal of the Mechanics and Physics of Solids, 44(6):1007-1032, 1996.

X. Zhou and K. K. Tamma. On the applicability and stress update formulations for corotational stress rate hypoelasticity constitutive models. Finite Elements in Analysis and Design, 39(8):783-816, May 2003. ISSN 0168874X. doi: 10.1016/S0168-874X(03)00059-3.

Yilin Zhu, Guozheng Kang, Qianhua Kan, and Otto T. Bruhns. Logarithmic stress rate based constitutive model for cyclic loading in finite plasticity. International Journal of Plasticity, 54:34-55, March 2014. ISSN 0749-6419. doi: 10.1016/j.ijplas.2013.08.004. 


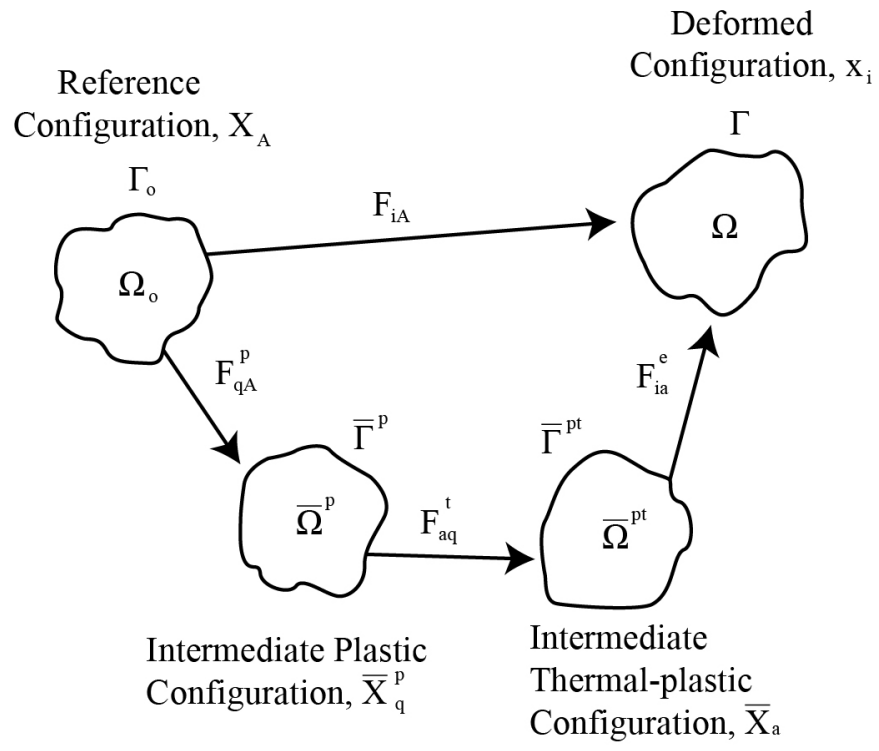

Fig. 1. Multiplicative Split of F per equation (1)

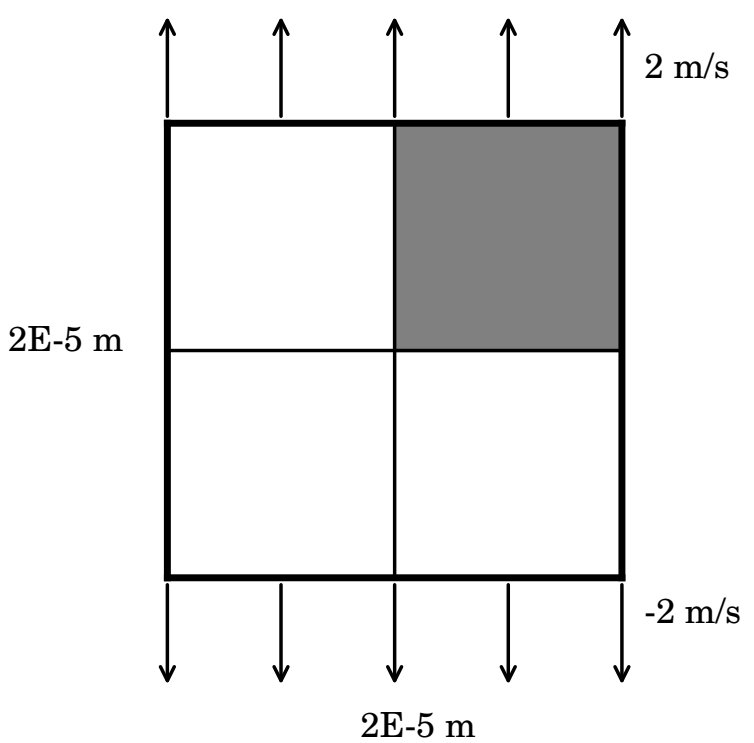

Fig. 2. Problem schematic for the tension problem. 


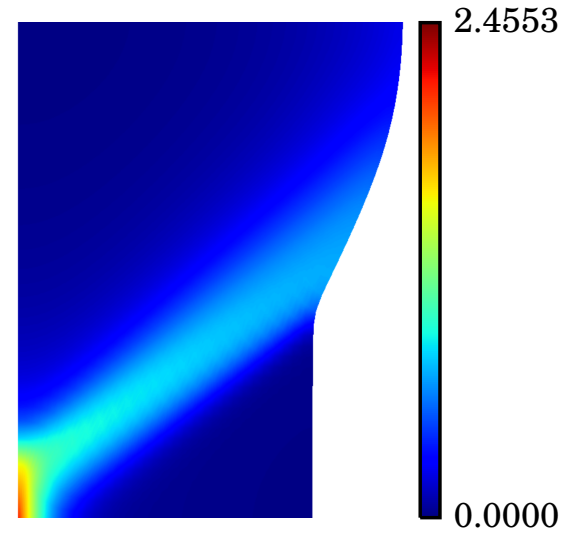

(a) Hyperelastic, $\mathrm{NH}$

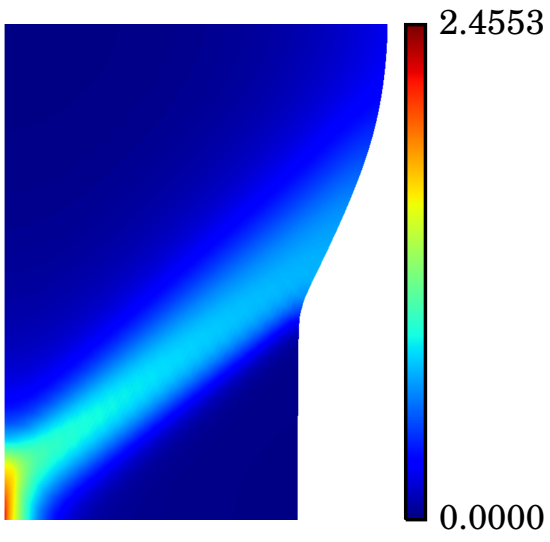

(b) Hyperelastic, HS

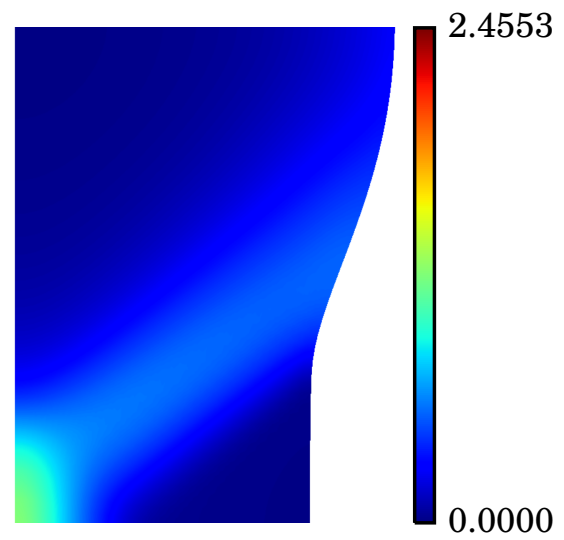

(c) Hypoelastic

Fig. 3. Surface plots of equivalent plastic strain for the Neo Hookean hyperelastic formulation (top left), Hencky strain hyperelasic formulation (top right), and the hypoelastic formulation (bottom) 
- Hyperelastic, $\mathrm{NH} \quad$ Hyperelastic, $\mathrm{HS} \quad$ Hypoelastic

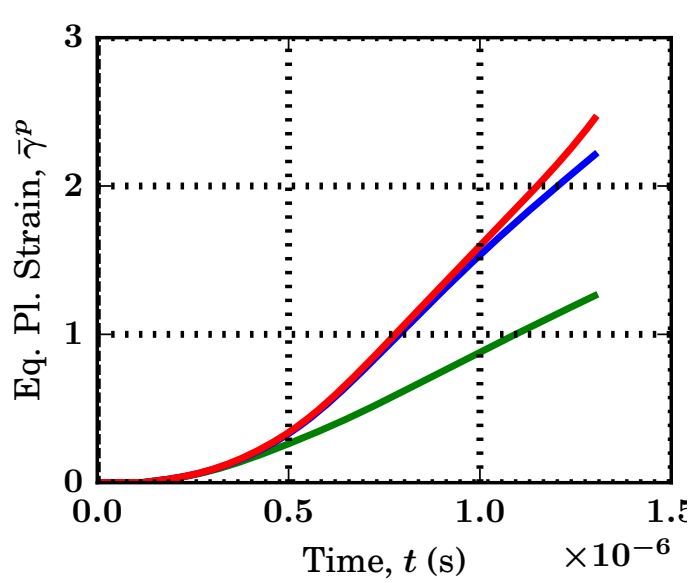

(a)

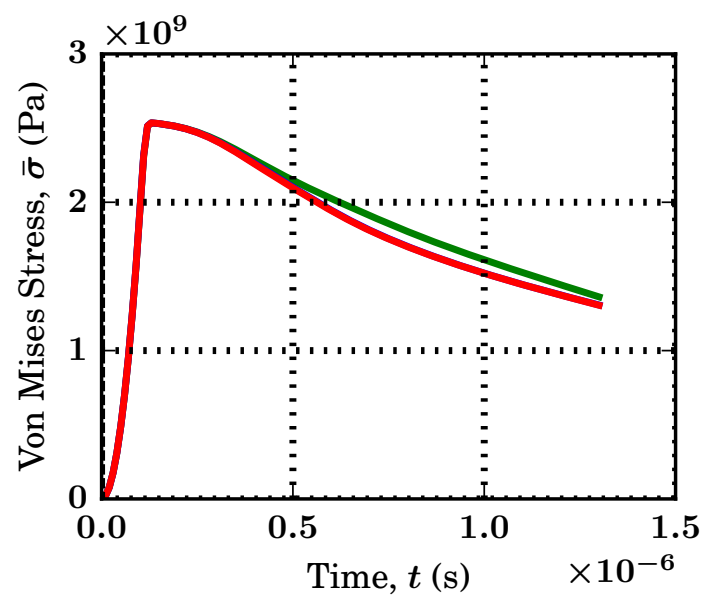

(b)

Fig. 4. Equivalent plastic strain vs time (left) and Von Mises stress vs time (right). After the plastic strains become greater than about 0.3 , the growth of plastic strain is significantly faster for the hyperelastic formulation. The stress strain curves are very close for the two formulations, and the two hyperelastic models plot on top of one another.

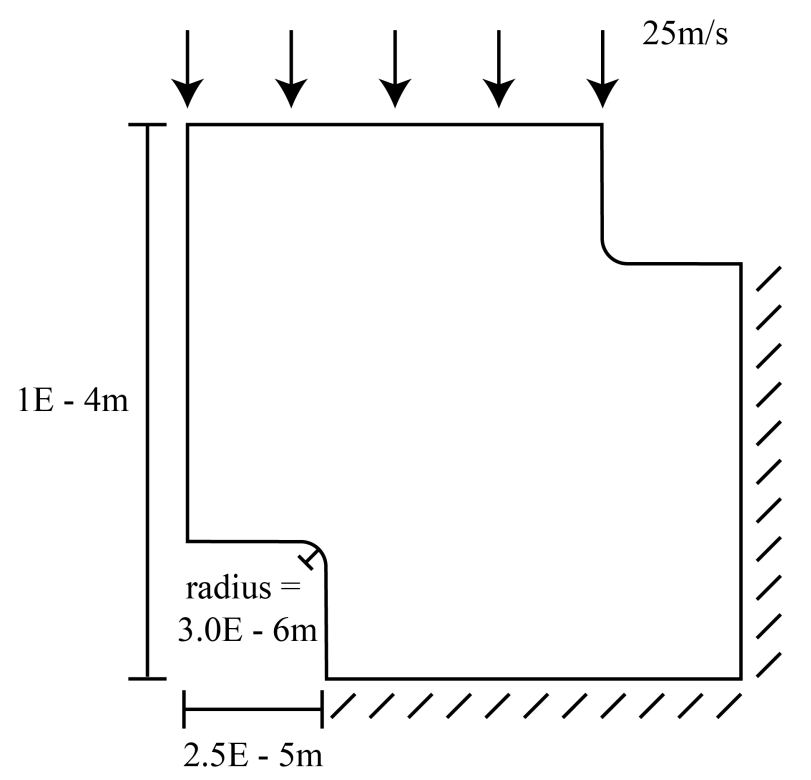

Fig. 5. Problem schematic for the compression problem. 


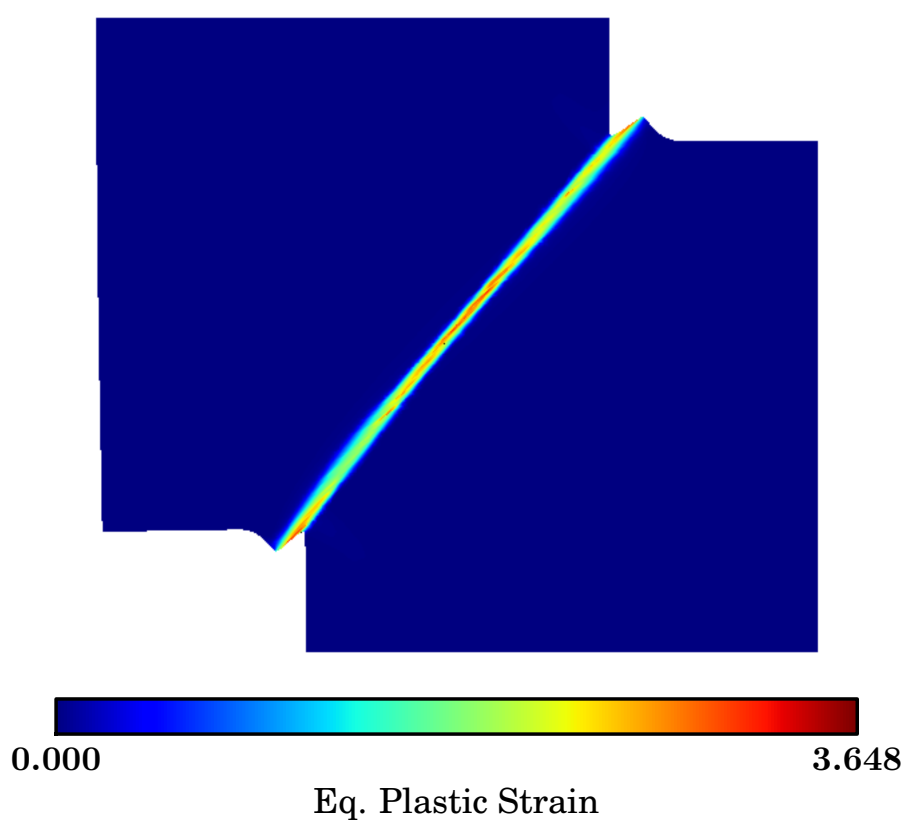

(a) Hyperelastic

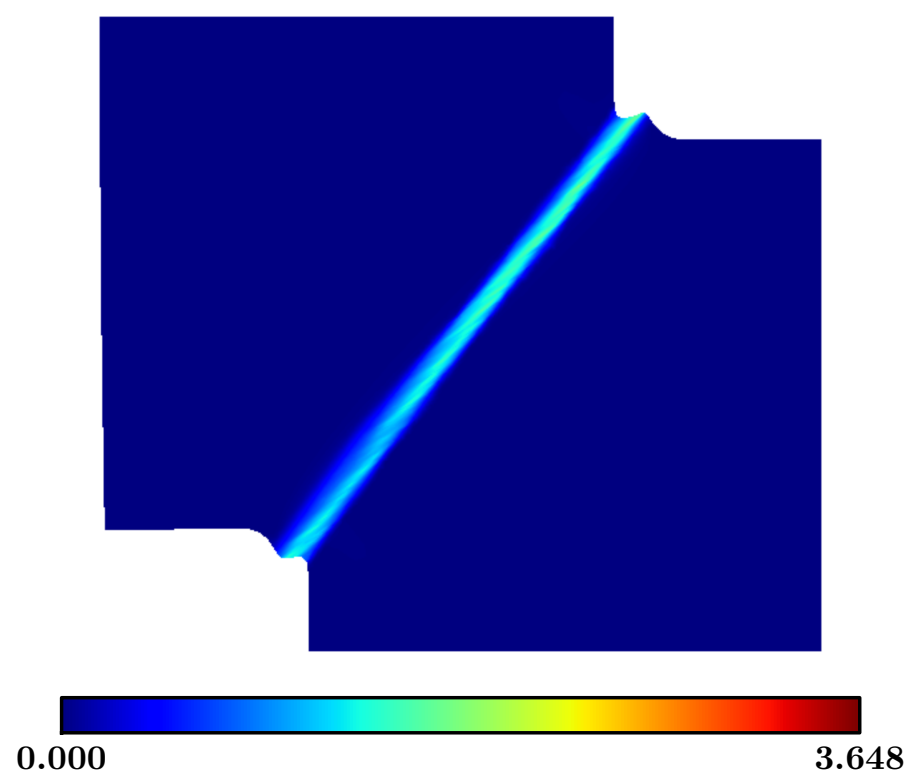

Eq. Plastic Strain

(b) Hypoelastic

Fig. 6. Surface plots of equivalent plastic strain for the hyperelastic formulation (top) and the hypoelastic formulation (bottom) at a time of $7.5 \mathrm{E}-7 \mathrm{~s}$ 


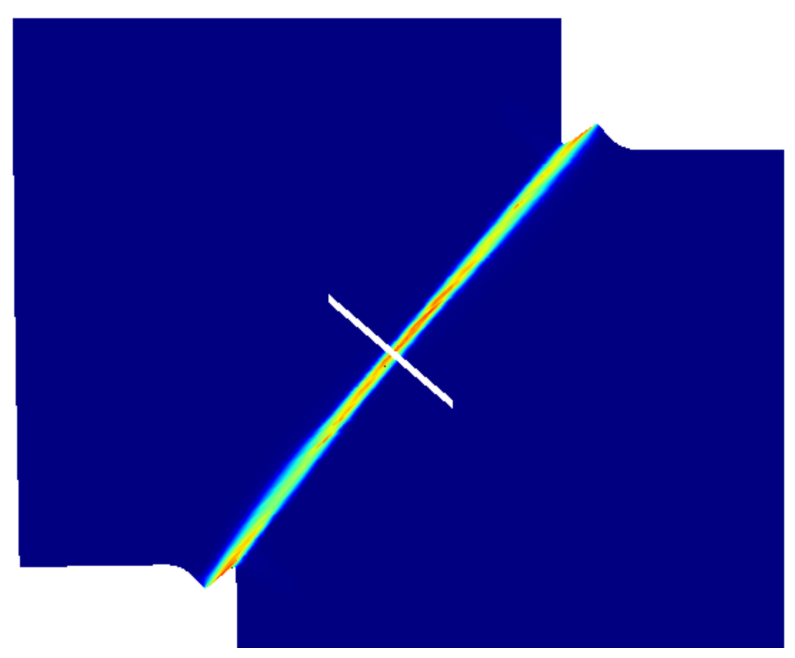

- Hyperelastic, $\mathrm{NH} \quad$ Hypoelastic

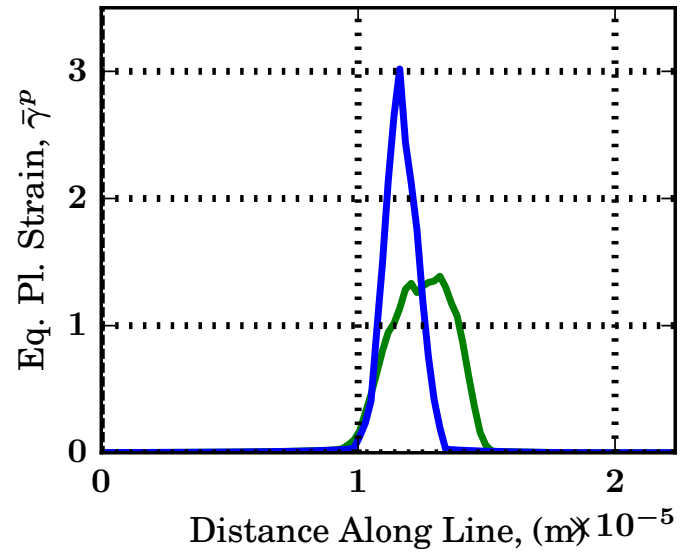

(a)

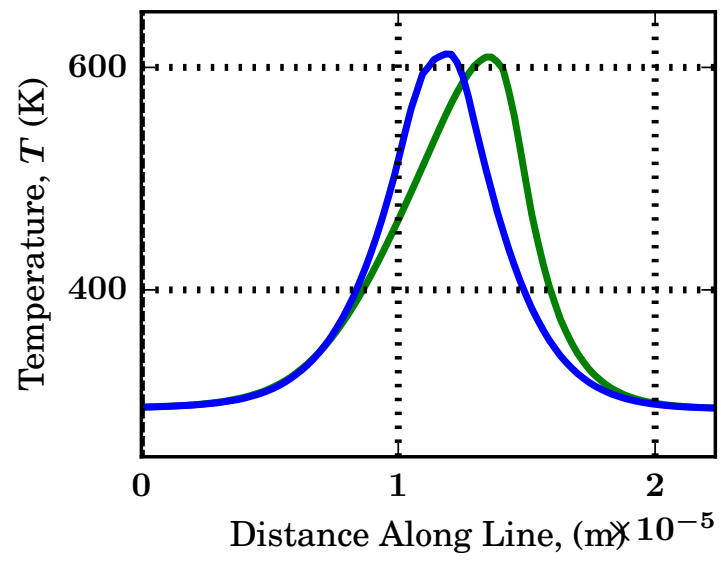

(b)

Fig. 7. Equivalent plastic strain (left) and temperature (right) distribution across the shear band at a time of 7.5E-7 s. These quantities are plotted over the line shown in white above. The peak temperatures are nearly identical for the two formulations, but the shear band is narrower and more intense for the hyperelastic formulation. 


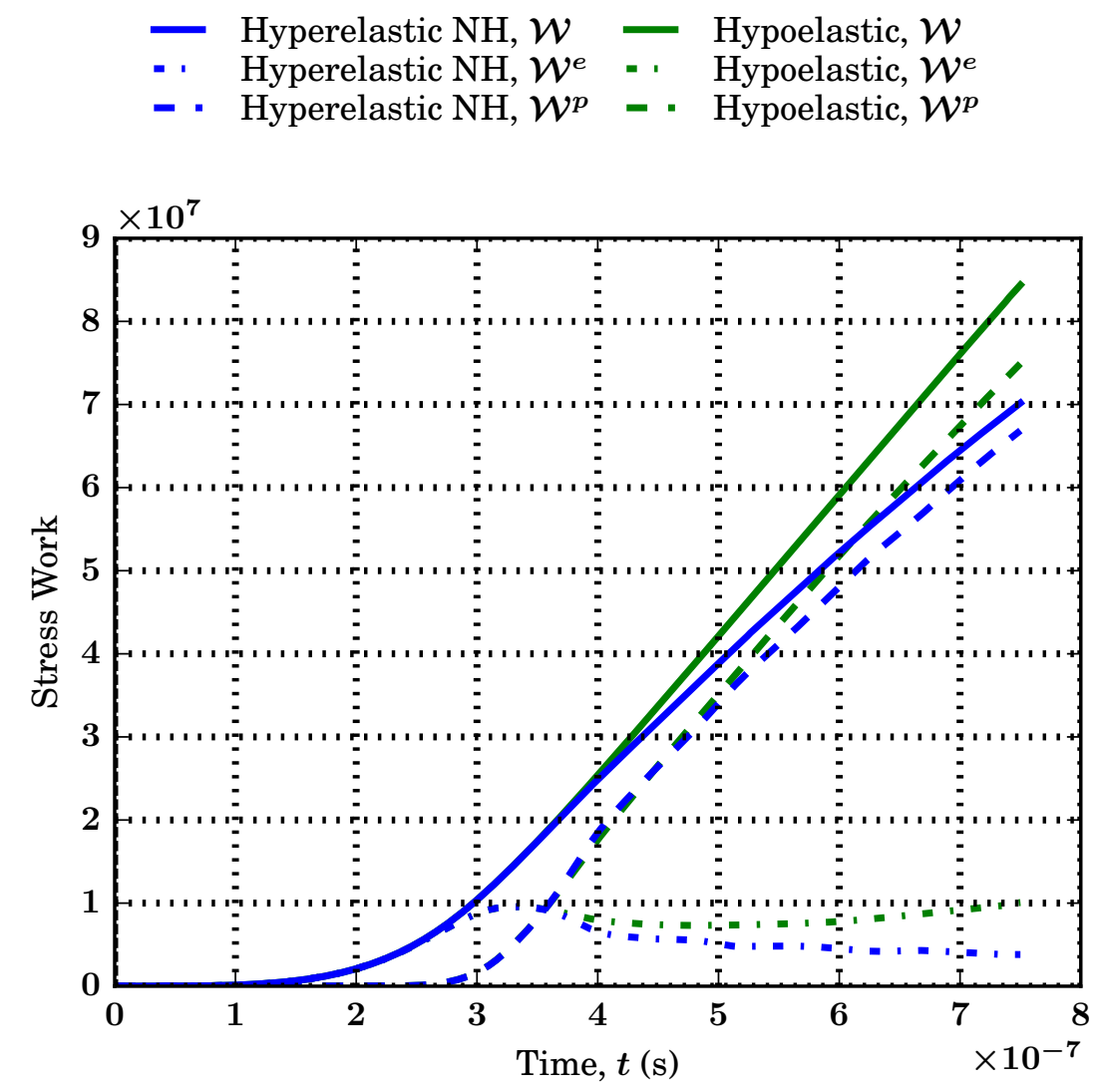

Fig. 8. Domain integrated mechanical work, showing the total as well as the elastic and inelastic contributions for the compression problem. These quantities are defines equations (97) - (100). 

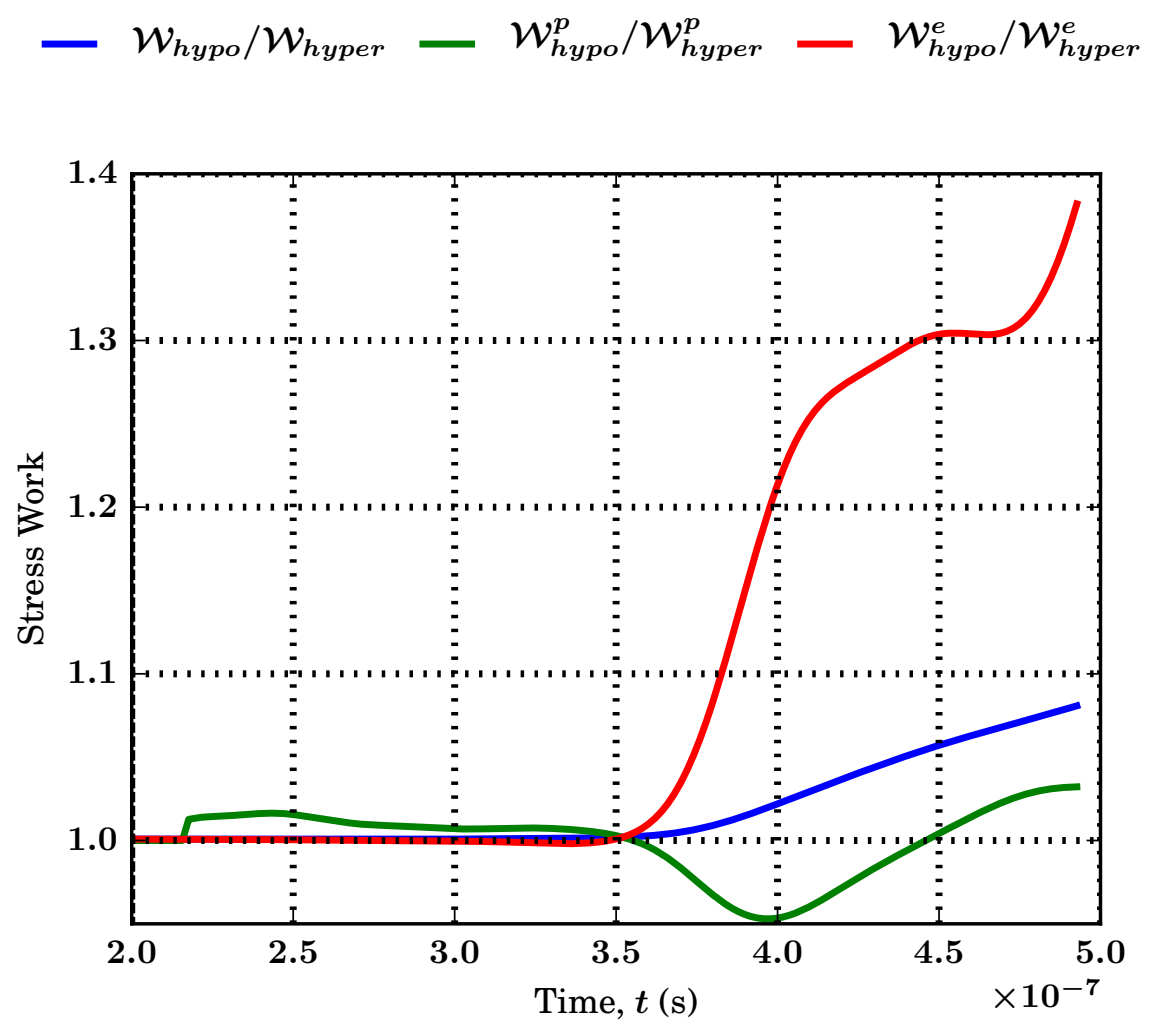

Fig. 9. Ratios of the mechanical work quantities for the two formulations, zoomed to the time interval of $2-5 \mathrm{e}-7$ seconds, the time range in which the two formulations begin to depart. 


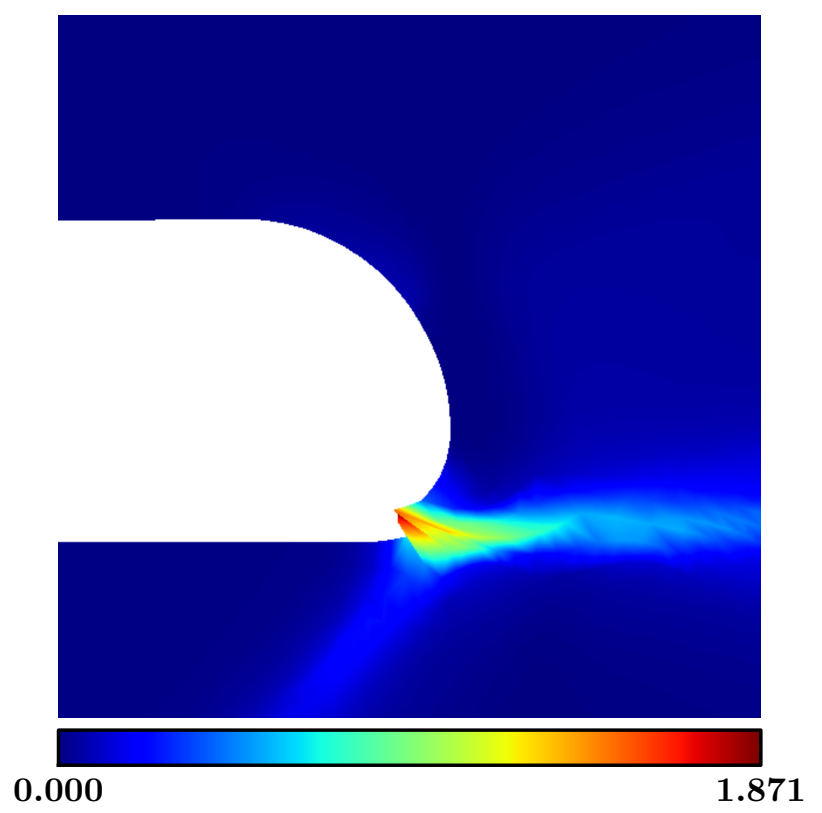

Eq. Plastic Strain

(a) Hyperelastic

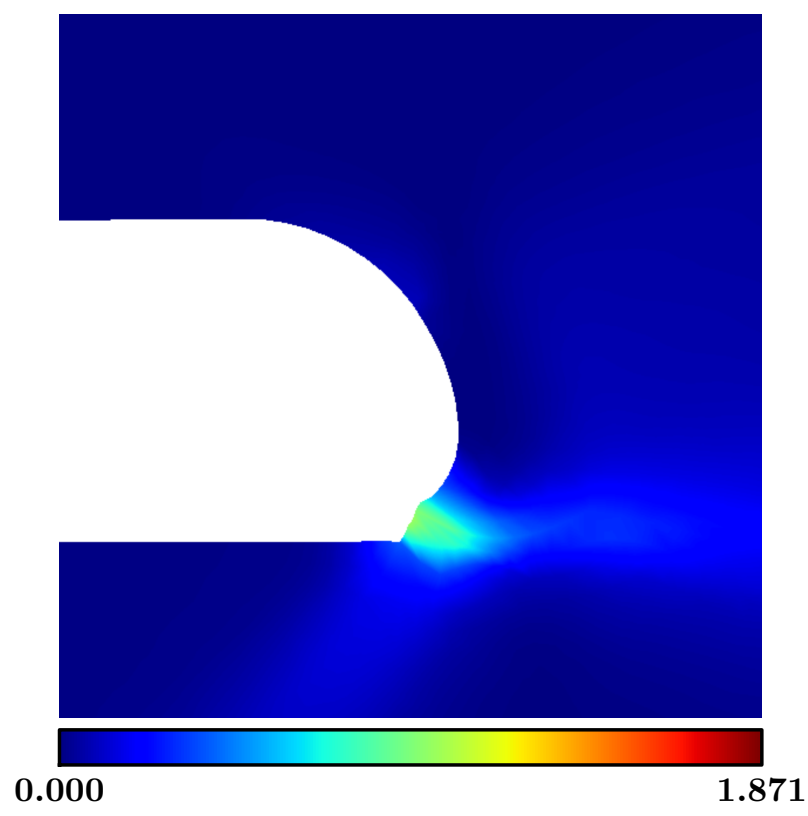

Eq. Plastic Strain

(b) Hypoelastic

Fig. 10. Surface plots of equivalent plastic strain for the hyperelastic formulation (left side) and the hypoelastic formulation (right side) at a time of 1.955E-05 
- Hyperelastic NH, $10 \mathrm{~m} / \mathrm{s}$ - , Hypoelastic, $10 \mathrm{~m} / \mathrm{s}$

- Hyperelastic NH, $20 \mathrm{~m} / \mathrm{s}$ - - Hypoelastic, $20 \mathrm{~m} / \mathrm{s}$

- Hyperelastic NH, $30 \mathrm{~m} / \mathrm{s}$ Hypoelastic, $30 \mathrm{~m} / \mathrm{s}$

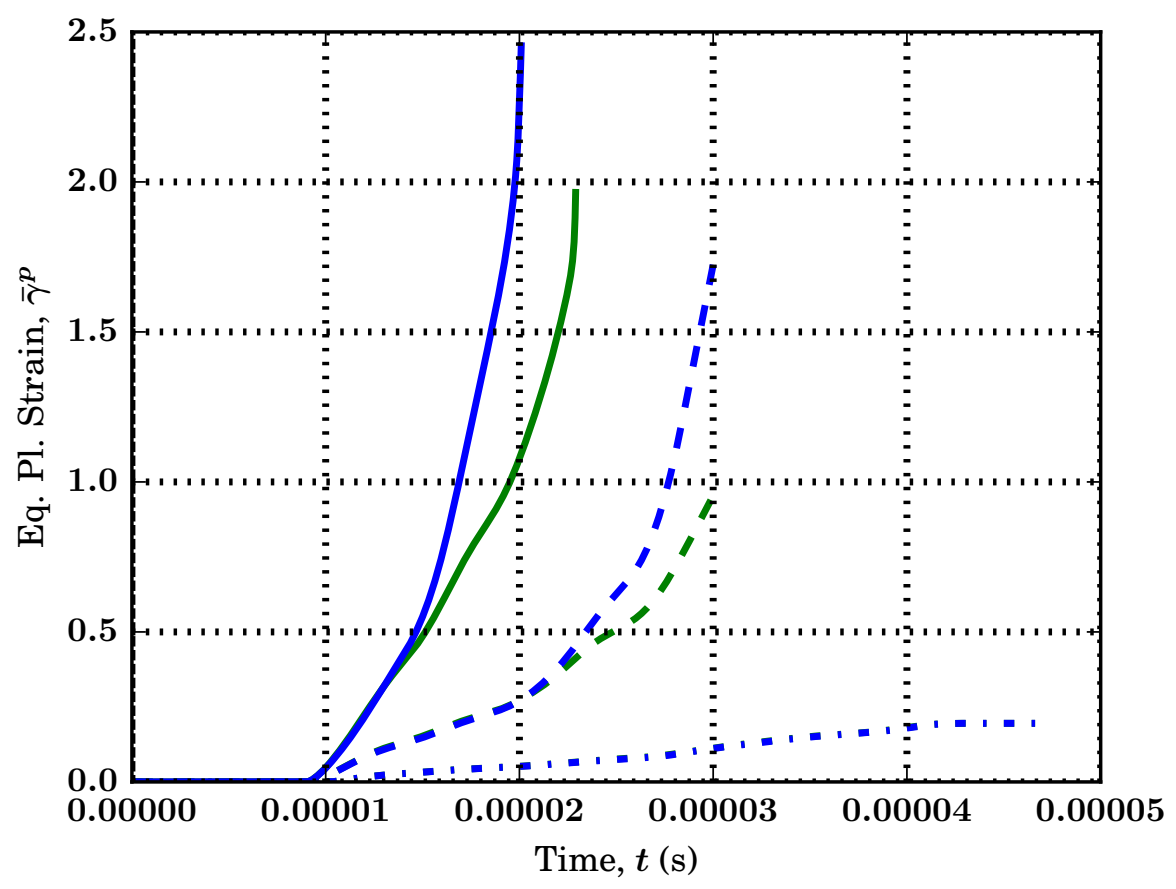

(a)

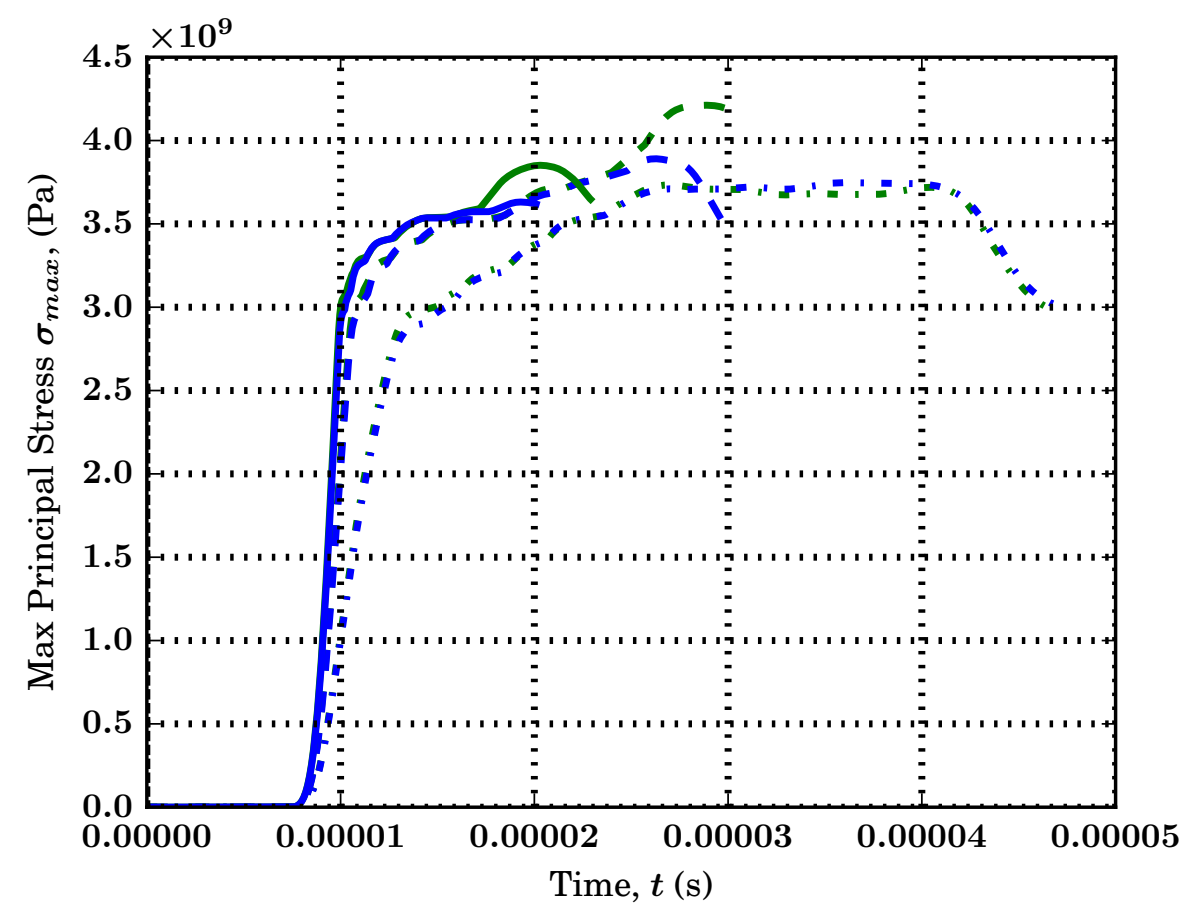

(b)

Fig. 11. Equivalent plastic strain vs time (a) at the shear band initiation point and the maximum principal stress vs time (b) for each of the formulations and impact velocities. The hyperelastic formulation results in earlier shear band initiation than the hypoelastic formulation for th $\$ 020$ and $30 \mathrm{~m} / \mathrm{s}$ impact velocities. For an impact velocity of $10 \mathrm{~m} / \mathrm{s}$, no shear band forms and the two formulations are not distinguishable. 